\title{
1 Size-independent mRNA synthesis and chromatin-based partitioning mechanisms 2 generate and maintain constant amounts of protein per cell
}

3

4 Matthew P. Swaffer ${ }^{1}$, Devon Chandler-Brown ${ }^{1}$, Maurice Langhinrichs ${ }^{1}$, Georgi Marinov ${ }^{2}$, William

5 Greenleaf $^{2}$, Anshul Kundaje $^{2}$, Kurt M. Schmoller ${ }^{1,3}$, Jan M. Skotheim ${ }^{1 *}$

6

$7 \quad{ }^{1}$ Department of Biology, Stanford University, Stanford CA, 94305, USA

$8 \quad{ }^{2}$ Department of Genetics, Stanford University, Stanford CA, 94305, USA

$9{ }^{3}$ Institute of Functional Epigenetics, Helmholtz Zentrum München, 85764 Neuherberg, Germany

11 * correspondence: skotheim@stanford.edu

\section{Summary}

15 Cell size and biosynthesis are inextricably linked. As cells grow, total protein synthesis increases in 16 proportion to cell size so that protein concentrations remain constant. As an exception, the budding 17 yeast cell-cycle inhibitor Whi5 is synthesized in a constant amount per cell cycle, so that it is diluted in 18 large cells to trigger division. Here, we show that this size-independent expression of Whi5 results 19 from size-independent transcription. A screen for similar genes identified histones as the major class 20 of size-independent transcripts during the cell cycle, consistent with histone synthesis being coupled

21 to genome content rather than cell size. However, during asymmetric division size-independent 22 transcription is insufficient for size-independent protein expression and chromatin-binding ensures 23 equal amounts of protein are partitioned to unequally sized cells to maintain size-independent protein 24 amounts. Thus, specific transcriptional and partitioning mechanisms determine size-independent 25 protein expression to control cell size. 
A striking feature of cell growth is that total protein and RNA amount per cell increase approximately in proportion to cell volume as a cell increases in size (Fig. 1A) (Fraser and Nurse, 1978, 1979). To achieve this coordinated scaling of macromolecules with cell size, larger cells have proportionally higher transcription and protein synthesis rates (Creanor and Mitchison, 1982; Elliott, 1983; Elliott and McLaughlin, 1979; Elliott et al., 1979; Padovan-Merhar et al., 2015; Sun et al., 2020; Zhurinsky et al., 2010). This size-scaling is generally important because it ensures macromolecule copy number is proportional to cell volume and therefore concentrations are kept constant as a cell grows (Fig. 1B) (Marguerat and Bahler, 2012; Neurohr et al., 2019). Nuclear volume also scales in proportion to cell volume meaning that nuclear concentrations are also expected to be constant (Jorgensen et al., 2007; Neumann and Nurse, 2007).

While it is generally assumed that most individual proteins exhibit this general size-scaling behavior and remain at constant concentration, one striking exception is the cell cycle inhibitor Whi5. Whi5 synthesis is uncoupled from cell size such that a constant amount of Whi5 is made in each cell division cycle independently of cell size, and biosynthetic capacity (Schmoller et al., 2015). Moreover, examination of Whi5 synthesis in a variety of extracellular growth conditions showed that a similar number of molecules were made in all conditions (Qu et al., 2019). This growth and size-independent synthesis pattern for Whi5 ensures its dilution in larger cells to trigger their division and thereby links the basic mechanisms of biosynthesis to cell size control. Thus, cell size determines the pattern of protein biosynthesis, which triggers division in larger cells to, in turn, control cell size. However, the underlying molecular mechanisms determining the relationship between cell size and the expression of individual proteins remain largely unknown.

In principle, any stage of gene expression could be regulated in a manner that results in sizeindependent protein synthesis (Fig. 1C). Importantly, this is not done using negative feedback because multiple copies of the WHI5 gene produces a proportional increase in the number of proteins made per cell cycle (Qu et al., 2019; Schmoller et al., 2015). To determine whether Whi5's size-independent behavior originates at the protein or transcript level, we sorted cells of different sizes using a total protein dye as proxy for cell size and performed RNA-seq (Fig. 1D \& S1A). Total mRNA content and cell volume were correlated with protein dye intensity confirming it as a good proxy for cell size (Fig. S1B-E). WHI5 mRNA transcripts per million (TPM) are anticorrelated with cell size, which implies that WHI5 mRNA concentration is lower in larger cells. In contrast, MDN1 mRNA TPM, as representative of scaling gene expression, was constant (Fig 1D). When normalized to total mRNA, using an external spike-in, WHI5 mRNA amount per cell was flat as a function of size (Fig. 1E-F). To corroborate this finding, we performed single-molecule FISH in individual cells while also measuring cell size (Fig 1G). Consistent with the RNA-seq data, WHI5 mRNA amounts were relatively independent of cell size whereas the MDN1 counts increased with cell size (Fig. 1H\&l, Fig S1A\&B). 
Because WHI5 mRNA is a cell cycle regulated transcript that peaks in S phase (Fig. S3C, (Pramila et al., 2006)) and cell size will partially correlate with cell cycle stage, it is possible the apparent size-independent behavior of WHI5 mRNA (Fig. 1E-H) is simply a consequence of the cell cycle rather than cell size per se. To control for this possibility, we isolated cells in early G1 by centrifugal elutriation and arrested them in $\mathrm{G} 1$ for increasing amounts of time to generate populations of cells of increasing sizes. Cells were then released from the G1 arrest resulting in cultures of cells synchronously traversing the entire cell cycle but at different sizes (Fig. 2A\&B \& Fig. S2). RNA-seq across the time-course allowed us to compare gene expression in cells of different sizes at precisely the same cell cycle stage (Fig. 2C). WHI5 TPM are indeed lower in larger cells as WHI5 expression peaks in S phase. The total amount of WHI5 expression across the entire cell cycle can then be estimated as the area under the curve, which again shows an anticorrelation between WHI5 mRNA concentration and cell size (Fig. 2D). Consistent with this, if we restrict our smFISH analysis to cells in early S/G2/M, when WHI5 expression peaks, the number of WHI5 transcripts are still uncorrelated with cell size (Fig. 2E \& S3E\&F). Taken together, this group of experiments suggests that WHI5 transcription or mRNA degradation is responsible for the size-independent expression of Whi5 protein.

Next, we sought to test if Whi5 size-independent expression originates at the level of transcription. If this were the case, then the WHI5 promoter should be both necessary and sufficient for size-independent protein expression. To test if the WHI5 promoter is sufficient, we compared the size-dependency of Whi5-mCitrine protein synthesis expressed from its endogenous promoter with that of a reporter mCitrine also expressed from the WHI5 promoter (Fig. 2F\&G). Both Whi5-mCitrine and the $\mathrm{mCitrine}$ reporter are synthesized in a size-independent manner. By contrast, when we expressed Whi5-mCitrine from a scaling promoter (ACT1pr) its synthesis rate now does increase with cell size (Fig. $2 \mathrm{H}$ ). Together, these experiments demonstrate that WHI5 is transcribed in a sizeindependent manner and that the WHI5 promoter is both necessary and sufficient for the sizeindependent synthesis of Whi5.

Having shown that size-independent expression of WHI5 is due to a transcriptional mechanism, we sought to determine which other cellular processes are similarly uncoupled from cell size. To do this we sought to determine which other sets of genes are similarly regulated by analyzing our RNA-seq experiments comparing cells of different sizes. We found 16 transcripts that behaved similarly to WHI5 in both experiments (Figure S4A). These genes are significantly enriched for GO terms related to chromatin and revealed histones as a major class of size-independent genes as 9 of the 16 identified genes encode histones (Fig. 3A). As for WHI5, histone mRNAs decreased in larger cells (Fig. 3B\&C and Fig. S2B). Again, this is not simply a consequence of their cell cycle regulated

94 transcription because the same trend was observed in cells of different sizes synchronously 95 progressing through the entire cell cycle (Fig. 3D\&E and Fig. S4C). We further confirmed the size96 independent expression of histone transcripts by comparing the mRNA levels in 1,484 strains 
containing a single gene deletion (Kemmeren et al., 2014; O'Duibhir et al., 2014) with the cell size of the respective gene deletion strain (Hoose et al., 2012; Jorgensen et al., 2002; Ohya et al., 2005; Soifer and Barkai, 2014). This revealed a clear anti-correlation between cell size and histone mRNA levels (Fig. 3F and S4D\&E).

The size-independent expression of histone mRNAs suggests that histone protein expression

102 is coordinated with genome content rather than cell size and predicts that histone protein synthesis 103 should also be size-independent. To examine this, we first analyzed two published datasets of flow 104 cytometry measurements across the collection of strains in which each individual open reading frame 105 was fused to GFP. We compared the relationship between GFP fluorescence (protein amount) and 106 side scatter (SSC-A, cell size) (Fig. 3G). This shows that histone proteins are less correlated with cell 107 size than the average protein in the proteome, i.e., the slope between cell size (SSC-A) and GFP 108 intensity is smaller (Fig. 3H\&l). To confirm that histone protein synthesis is indeed size-independent, 109 we quantified the amount of histone synthesized across the cell cycle of single cells by time-lapse 110 fluorescence microscopy and compared it with the increase in size during the same period. Cells that 111 grow more produce proportionally more of a scaling protein (Rpb3), but less histones (Hta2, $\mathrm{Htb} 2$ and $112 \mathrm{Htz1}$ ) (Fig. 3J\&K). Taken together these experiments have identified histones as a rare class of size113 independent genes whose transcription, and therefore protein synthesis, is uncoupled from cell size.

114 In this way, histone production is coordinated with genome-content rather than cellular growth.

115 Both Whi5 and histones are stable proteins synthesized in a size-independent amount during $116 \mathrm{~S} / \mathrm{G} 2 / \mathrm{M}$ of the cell cycle meaning that their amounts in $\mathrm{G} 1$ are determined by inheritance from previous 117 cell cycles. The asymmetric division of budding yeast poses a problem for maintaining size118 independent amounts of these proteins because the smaller daughter cells would inherit fewer such 119 proteins if they were partitioned in proportion to cell volume as is generally the case across the 120 proteome. For volume-based partitioning, concentrations are expected to be similar in the mother and 121 daughter cells following division as seen for a freely diffusing mCitrine (Fig. 4A). However, this is not 122 the case for Whi5, which we find is not partitioned evenly by volume so that daughter cells all inherit a 123 more similar number of molecules (Fig. 4A). To perfectly maintain size-independent amounts, a 124 mechanism partitioning equal amounts to the daughter and mother cells is required.

125 To calculate the impact of amount- and volume-based partitioning modalities on the amounts 126 of inherited protein, we employed a full cell-cycle model that simulates growth and division of a 127 population of cells. This model was parameterized by single-cell microscopy measurements and 128 therefore accounts for cell-to-cell variability and the size-dependence of cell cycle progression 129 (Chandler-Brown et al., 2017). To this model, we added a protein synthesized either at a rate 130 proportional to cell size or independent of size. At division, these proteins were either partitioned in 131 proportion to cell-volume or in the manner empirically determined for Whi5 where a significant fraction 132 is partitioned by amount (Fig. 4B, S6A\&B). Our simulations show that the amount of Whi5 inherited in 
133 G1 should be significantly less size-independent if it were partitioned by cell volume rather than by 134 protein amount. This is in part because bud size varies significantly even for mothers with same the 135 volume. Together this suggests that both partitioning by amount and size-independent synthesis 136 ensures the size-independent amount of Whi5 in G1.

One possibility to generate amount-based partitioning of Whi5 would be to harness the equal 138 partitioning of genome segregation. This hypothesis was suggested by the fact that Whi5 binds the 139 DNA-bound SBF transcription factor complex following its dephosphorylation at mitotic exit. To test 140 this, we analyzed the partitioning of a Whi5 variant (Whi5WIQ) that does not bind SBF and is not 141 recruited to the SBF binding sites in the CLN2 or SVS1 promoter (Travesa et al., 2013). First, we 142 confirmed that the WIQ mutation reduces Whi5 binding at SBF bound DNA elements across the 143 genome by ChIP-seq (Fig. S6A). Next, we analyzed cells expressing Whi5(WIQ)-mCitrine, which 144 revealed it has a significantly lower bud-to-mother concentration ratio at division than wild-type Whi5. 145 This supports our model that partitioning by amount is indeed mediated by DNA-binding (Fig. 4A). 146 Crucially, Whi5(WIQ) amounts at birth are higher in larger cells and lower in smaller cells when 147 compared to wild-type Whi5, demonstrating that when Whi5 partitioning is disrupted, its size148 independence in G1 is also disrupted (Fig. 4C\&D). Thus, our data support a model where Whi5 binding 149 to chromatin results in its volume-independent partitioning into the mother and bud at division to ensure 150 daughter cells inherit approximately the same amount of Whi5 regardless of their size.

In conclusion, while most proteins are synthesized so that their amounts are proportional to 152 cell size and their concentrations remain constant, some are not (Fig. 1A\&B). We previously identified 153 the cell cycle inhibitor Whi5 and its functional ortholog in human cells, the retinoblastoma protein, as 154 proteins whose amount synthesized per cell is independent of cell size (Schmoller et al., 2015; 155 Zatulovskiy et al., 2020). This results in smaller cells having proportionally higher concentrations of 156 these cell cycle inhibitors to promote more growth in $\mathrm{G} 1$ to compensate their initially smaller birth size. 157 Here, we show that in budding yeast this is achieved via a transcriptional mechanism and our 158 transcriptomics experiments have revealed that the G1/S cell cycle inhibitors are not alone. Strikingly, 159 the histone mRNAs constitute the majority of transcripts in this small group of genes whose expression 160 is size-independent. The size-independent expression of histones allows the maintenance of a 161 stoichiometric relationship between histone proteins and the genome without engaging the potentially 162 wasteful feedback mechanisms, which are known to operate when histone expression is artificially 163 elevated (Cross and Smith, 1988; Gunjan and Verreault, 2003; Moran et al., 1990; Norris and Osley, 164 1987).

165 For both histones and WHI5, size-independent amounts of protein are reflected in the mRNA amounts synthesized during the cell cycle. Our data from cycling cells mirrors the recent finding that 167 in cells arrested in G1 the mRNA concentration of a number of cell cycle activators increases in larger 168 G1 cells (Chen et al., 2020). Such a size-dependent concentration increase was first found in fission 
169 yeast for the cell cycle activator cdc25 (Keifenheim et al., 2017). However, understanding the

170 mechanisms controlling the various size-dependencies of transcription is limited by the fact we do not

171 understand how cell size controls transcription even in the generic size-dependent case where global

172 transcription rates increase in proportion to cell size (Padovan-Merhar et al., 2015; Sun et al., 2020;

173 Zhurinsky et al., 2010). Nevertheless, despite these limitations, our WHI5 promoter-swap experiments

174 are informative and suggest that specific promoter elements, with dedicated molecular mechanisms

175 to circumvent the global transcriptional increase, are at least in part responsible for size-independent

176 gene expression.

177 Here, we found that size-independent gene expression, where a constant number of molecules

178 is made per cell cycle, is insufficient to guarantee that a constant number of molecules are inherited

179 in newborn daughter cells. This problem is most acute when there is a major size asymmetry in

180 cytokinesis such as in the case in budding yeast but also metazoan cells such as D. melanogaster

181 neuroblasts or the early embryonic divisions of C. elegans (Chia et al., 2008; Sulston et al., 1983). In

182 these cases, if proteins synthesized in constant amounts per cell cycle were simply partitioned in

183 proportion to the relative volumes of the newborn cells, the size-independence would be lost. To

184 maintain size-independent protein expression following division, cells can use chromatin-based

185 partitioning mechanisms that harness the faithful segregation of sister chromosomes to partition near

186 equal amounts to the two newborn cells (Fig. 5). While it has long been appreciated that big and small

187 cells both have the same amount of DNA, we identified here a set of genes that are similarly size-

188 independent. Size-independent synthesis is reflected in the function of many size-independent genes

189 such as the histones, which require stoichiometry with the genome. It is both curious and elegant that

190 the size-independent gene set includes WHI5 which regulates DNA replication, while the DNA itself is

191 used as a scaffold for the synthesis and maintenance of size-independent gene expression. 
A

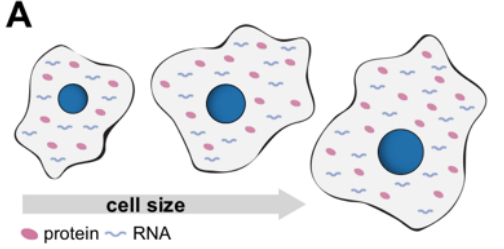

B

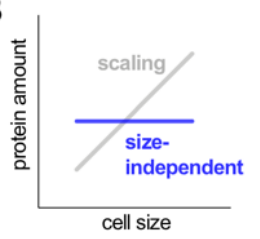

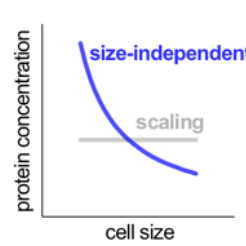

cell size

C possible origins of size-independent expression

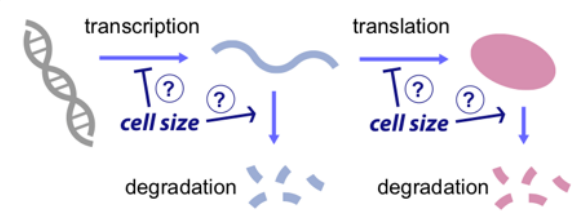

$\mathbf{F}$
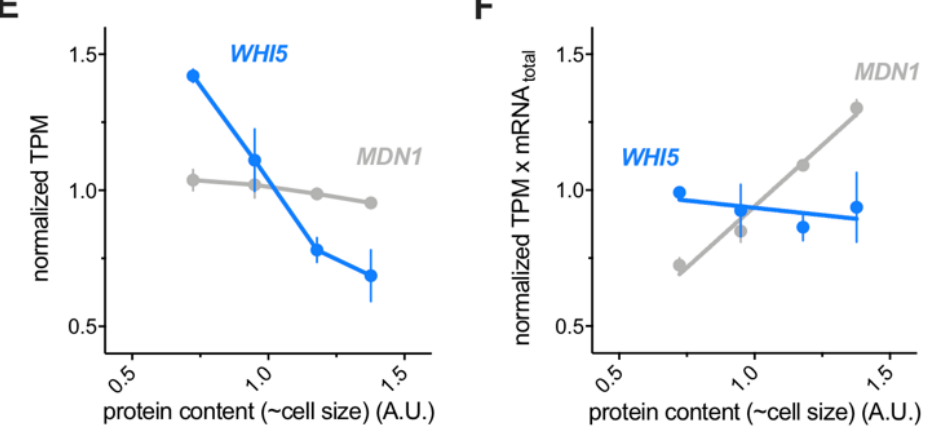

G

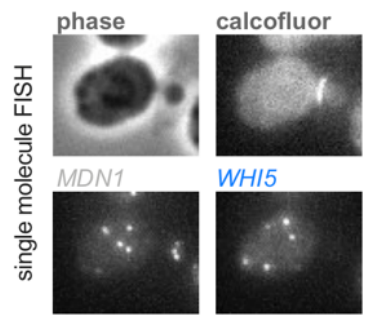

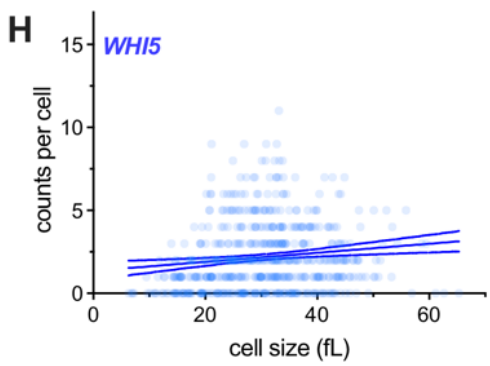

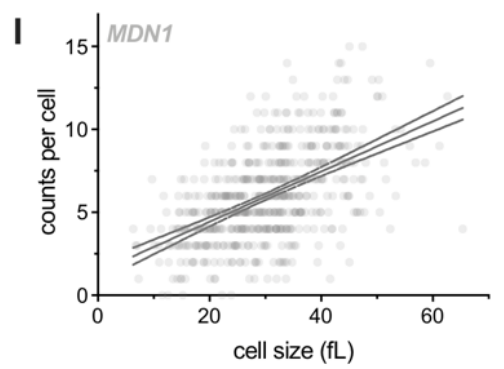

Figure 1 | WHI5 mRNA concentrations are uncorrelated with cell size

194 (A-C) schematics illustrating gene expression in proportion to or independent of cell size. (A) Total protein and RNA copy numbers per cell generally increase in proportion to cell volume so that their concentrations remain constant during growth. (B) However, some proteins are expressed at constant amounts independent of cell size and are diluted by cell growth, which (C) could result from regulation at any step of gene expression.

(D-F) Cells in S/G2/M were sorted into four bins based on the intensity of total protein dye. See Fig. S1 and Materials and Methods for details. (D) Histogram of total protein content per cell in each bin measured after sorting. (E) Normalized Transcripts Per Million (TPM / mean TPM) for WHI5 and MDN1 mRNA in cells of different sizes (total protein content). The mean (+/- range) of two biological replicates is plotted. Changes in TPM are proportional to changes in mRNA concentration. (F) Normalized TPM $x$ total-mRNA for WHI5 and MDN1 mRNA in cells of different sizes (total protein content). Mean (+/- range) of two biological replicates is plotted. Changes in TPM $x$ total mRNA are proportional to changes in mRNA amount. Relative total mRNA per cell was determined by the number of reads relative to those from a fixed number of $S$. pombe cells added to the sample.

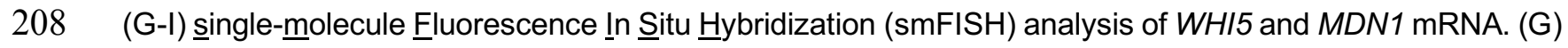
representative smFISH images. (H\&I) mRNA counts per cell as a function of cell size for WHI5 and MDN1 determined by smFISH, $n=567$ cells. Linear regression (solid line) and 95\% confidence interval (dashed lines) are shown. Data are pooled from two biological replicates. The same data with replicates plotted independently are shown in Fig. S3A\&B. 

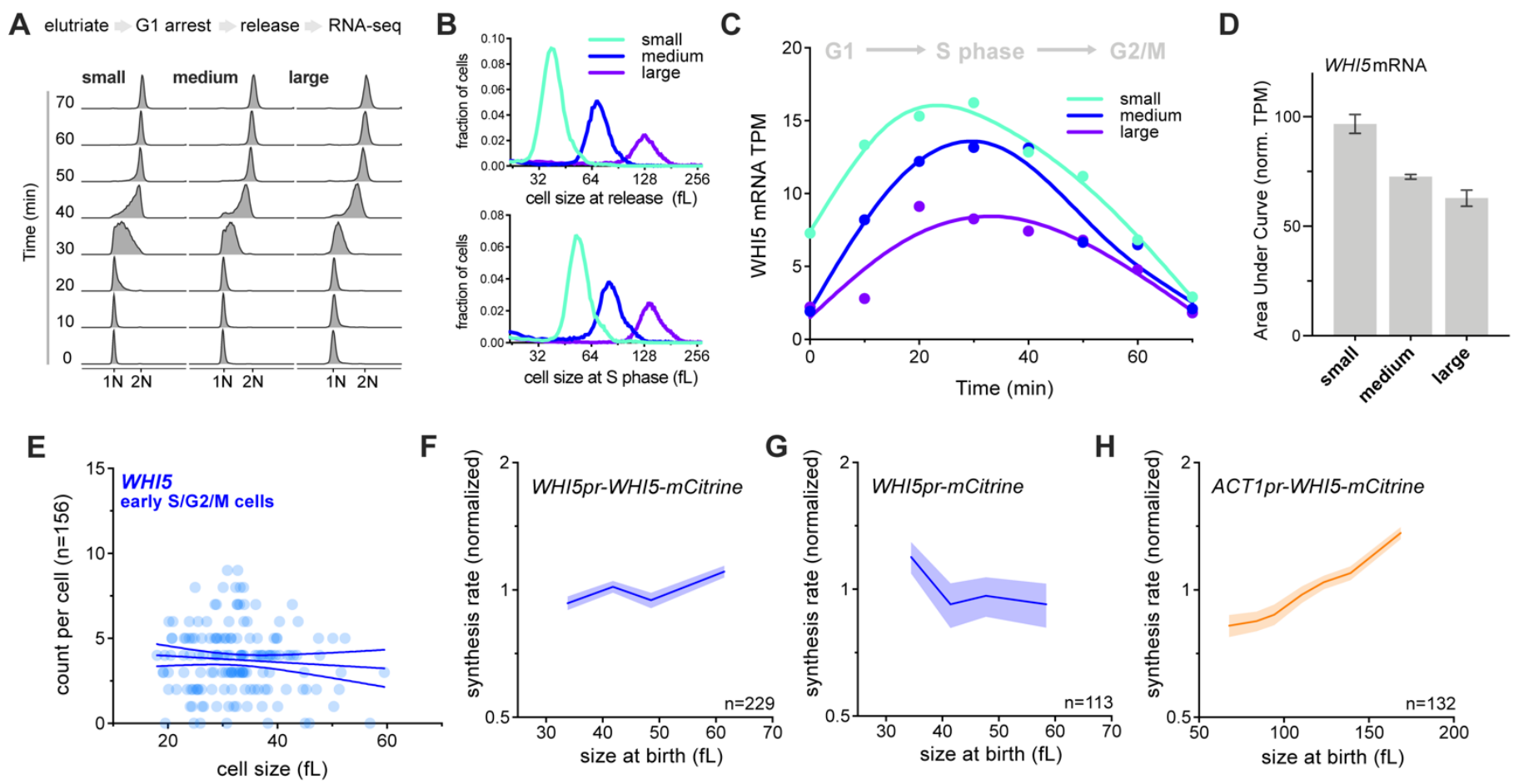

213

214 Figure 2 | WHI5 transcription is independent of cell size across the cell cycle

215 (A-D) G1 cells of different sizes (small, medium or large) were arrested for increasing amounts of time in

$216 \mathrm{G} 1$ using a temperature sensitive $c d c 28-13$ allele at $37^{\circ} \mathrm{C}$. Cells were then released from $\mathrm{G} 1$ to progress

217 synchronously through the cell cycle and analyzed by RNA-seq across the full cell cycle. See Fig. S2 for

218 details. (A) DNA content analysis determined by flow cytometry of small, medium and large as they

219 trasverse the cell cycle. (B) Size distribution of small, medium or large cells at point of release from G1

220 arrest (top panel) and at mid S-phase (bottom panel, corresponds to the 40 minute time point). (C) WHI5

221 mRNA TPM for small, medium or large cells synchronously progressing through the cell cycle. (D) The Area

222 Under the $\underline{\text { Curve }}$ (AUC) of mean normalized WHI5 mRNA TPM for small, medium or large cells

223 synchronously progressing through the cell cycle. The AUC mean (+/- range) of two biological replicates is

224 plotted.

225 (E) mRNA counts per cell for WHI5 as a function of cell size in early S/G2/M cells determined by smFISH,

$226 \mathrm{n}=156$ cells. Early S/G2/M cells were defined as budded cells with a small ( $\leq 0.2)$ bud-to-mother volume

227 ratio. Linear regression (solid line) and 95\% confidence interval (dashed lines) are shown. Data are pooled

228 from two biological replicates. The same data with replicates plotted independently, including data for

$229 M D N 1$, are shown in Fig. S3E\&F.

230 (F-H) Protein synthesis rates (normalized to the mean) as a function of cell volume at budding, measured

231 by time-lapse fluorescence microscopy measuring Whi5-mCitrine expressed from $(F)$ the endogenous

232 WHI5 promoter or $(\mathrm{H})$ the ACT1 promoter, and $(\mathrm{G})$ mCitrine expressed alone from the WHI5 promoter.

233 Synthesis rates were determined as in (Schmoller et al., 2015) for single cells using linear fits of total protein

234 traces for the period between bud emergence and cytokinesis (S/G2/M). Data are binned according to cell

235 size at budding and the mean (+/- SEM) of each bin is plotted. Un-binned single-cell values of the same

236 data are plotted in Fig. S2G-I. 


\section{A}

Gene ontology term enrichment

릉 Nucleosome $(p=5.37 E-22)$

Chromatin assembly or disassembly $(p=2.26 E-13)$

DNA binding $(p=5.7 E-8)$

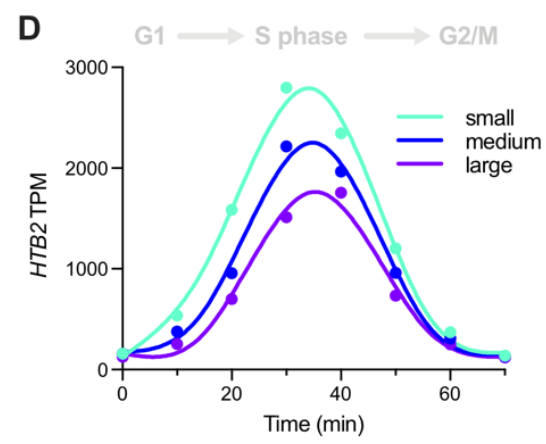

$E$

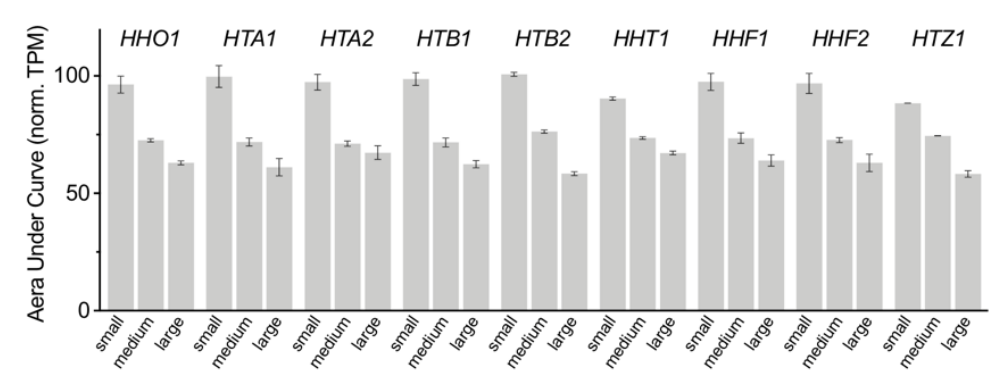

B

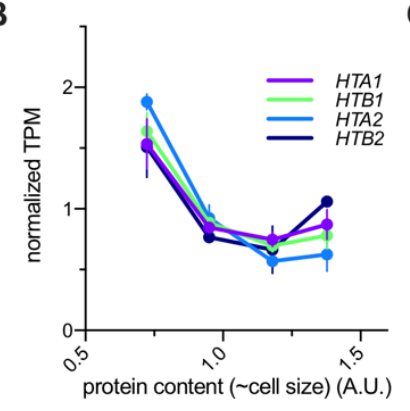

C

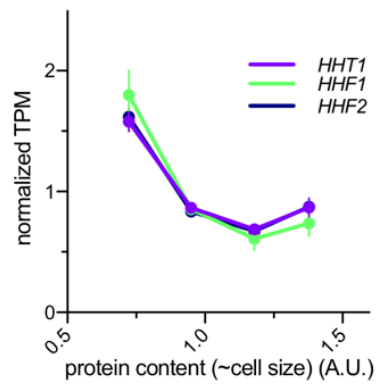

$\mathrm{E}$
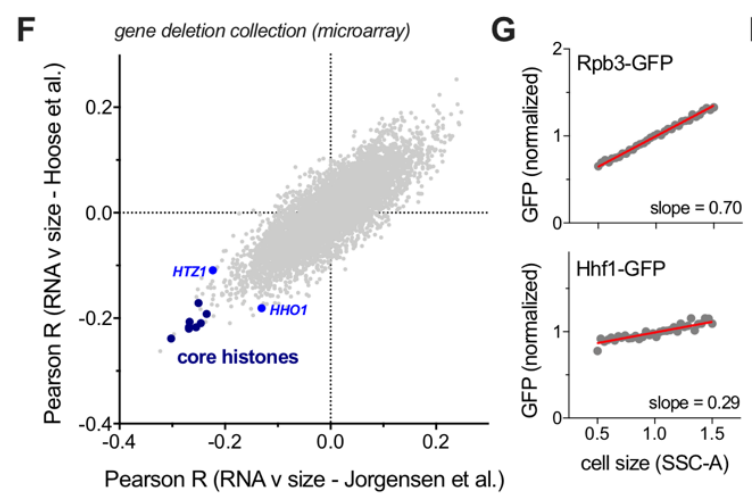

H GFPfusion collection (flow cytomety) I

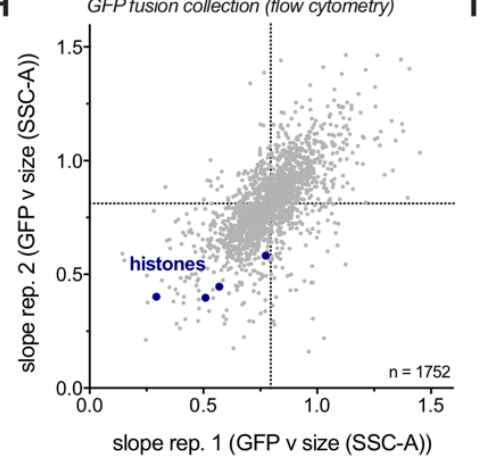

I

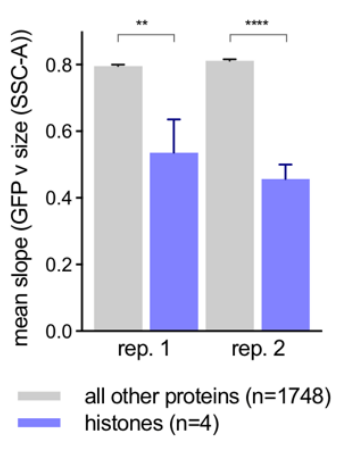

$\mathbf{J}$

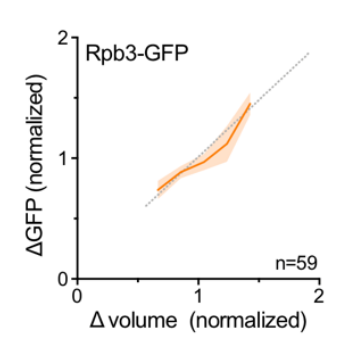

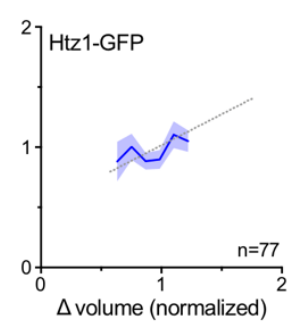

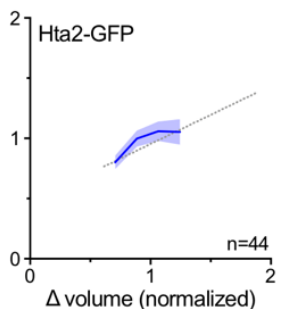

$\Delta$ volume (normalized)

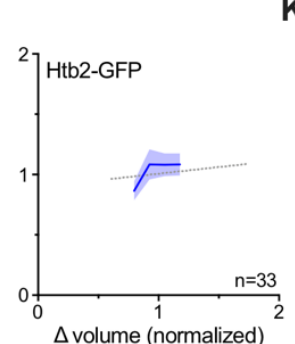

$\Delta$ volume (normalized)

Figure 3 | Histones are a rare class of size-independent genes

(A) Gene ontology terms enriched in size-independent genes. 9 of the 17 size-independent genes encode histones and 1 is WHI5. See Fig. S4A and Materials and Methods for classification details. (B\&C) Normalized TPM (TPM / mean TPM) for size-independent histone mRNAs in cells of different sizes (total protein content). The mean (+/- range) of two biological replicates is plotted. Changes in TPM are proportional to changes in mRNA concentration. See Fig. S1 for experimental details.

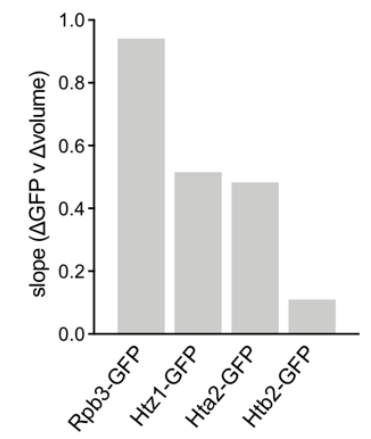


244 (D) HTB2 mRNA TPM for small, medium, and large cells synchronously progressing through the cell cycle 245 as in Fig.1J\&K. See Fig. S2 for experimental details.

246 (E) The Area Under the Curve (AUC) of mean normalized size-independent histone mRNA TPM of small, 247 medium, and large cells synchronously progressing through the cell cycle. The AUC mean (+/- range) of 248 two biological replicates is plotted.

249 (F) Pearson R correlation coefficient for the correlation between mRNA levels relative to wild-type in 1,484 250 gene deletion strains (Kemmeren et al., 2014; O'Duibhir et al., 2014) and the cell size of the respective 251 gene deletions for two different data sets of size measurements (Jorgensen et al. 2002 (x-axis), Hoose et 252 al. 2012 (y-axis)). Each point represents an individual gene. Histone mRNAs are shown in blue.

253 (G-I) Flow-cytometry analysis of size-dependent expression in the genome-wide collection of GFP fusion 254 strains. Side scatter (SCC-A) was used as a proxy for cell size. The slope of the linear fit between cell size 255 (SCC-A) and GFP intensity in budded cells was used to estimate the degree of size-dependence for each 256 protein. See Materials and Methods for details.

257 (G) Plot of example protein-GFP levels (intensities, normalized to the mean intensity) against cell size 258 (SCC-A, normalized to the mean SCC-A) in budded cells. Grey dots are bin means after binning on cell 259 size. Red lines show the linear regression to the un-binned data.

260 (H) Slope values (for the linear regression between GFP and cell size in budded cells) of 1752 proteins 261 analyzed in two replicates. Slopes closer to 0 correspond to more size-independent behavior. Histone 262 proteins are shown in blue.

263 (I) Average slope values (for the linear regression between GFP and cell size in budded cells) for histones 264 (blue) and all other proteins (grey). Four histones proteins were present in the 1752 proteins analyzed. 265 Histone proteins have significantly smaller slopes than the average protein (replicate 1: $p=0.0014$, replicate 266 2: $\mathrm{p}<0.0001)$.

267 (J) The amount of Rpb3-GFP (RNA polymerase II subunit) and three histones (Hta2-GFP, Htb2-GFP and 268 Htz1-GFP) synthesized ( $\triangle$ GFP, normalized to its mean) between birth and division as a function of the 269 amount of growth ( $\Delta$ volume, normalized to its mean), determined by single cell time-lapse fluorescence 270 microscopy. Data are binned according to $\Delta$ volume and the bin means (+/- SEM) are plotted. Robust linear 271 fit to un-binned data are shown (dashed grey line).

272 (K) Slope of the robust linear fits to $\Delta$ GFP against $\Delta$ volume as in (J). 
A

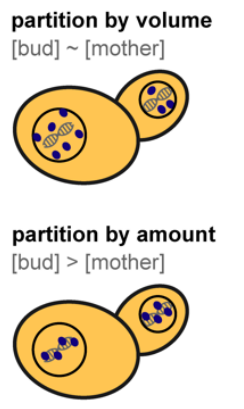

C

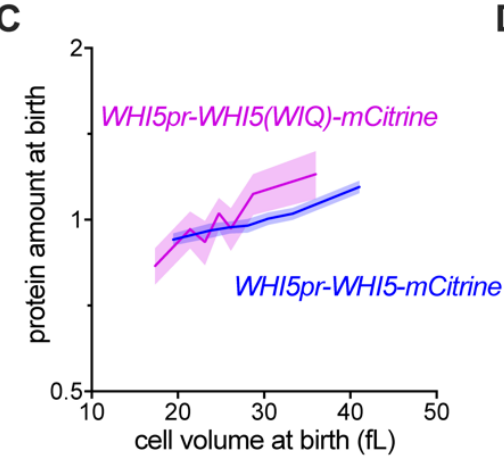

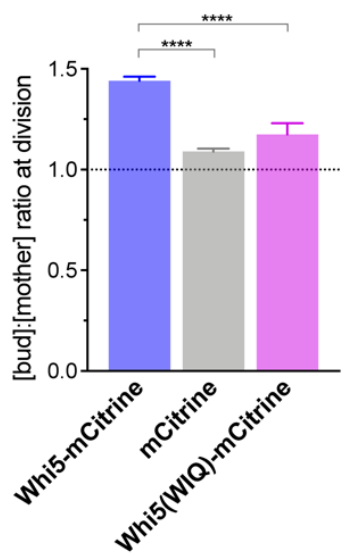

B

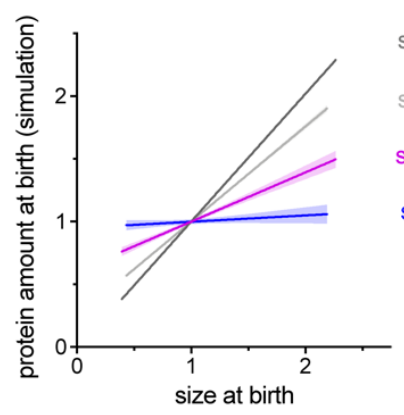

D

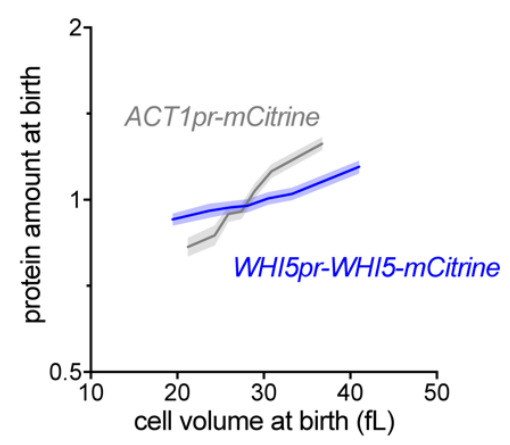

Figure 4 | Size-independent expression is inherited through asymmetric division due to DNAmediated partitioning

(A) The bud-to-mother concentration ratios for Whi5-mCitrine, free mCitrine or Whi5(WIQ)-mCitrine at cytokinesis. Whi5(WIQ)-mCitrine has reduced recruitment to DNA because it does not bind and inhibit the SBF transcription factor (Travesa et al., 2013) (Fig. S6A). A ratio $\sim 1$ is expected for proteins that are partitioned in proportion to volume. A ratio $>1$ is expected for proteins that are partitioned by protein amount (i.e., independent of the mother and bud volume). ${ }^{* * *} p<0.0001$.

(B) Simulation of protein amount at birth as a function of daughter volume at birth. Four different conditions were simulated: proteins expression is either size-dependent or size-independent, and protein partitioning is either by protein amount or by cell volume. Linear fit to simulated cell size and protein amounts at birth are shown. Raw simulated values are plotted in Fig. S6B\&C. See Materials and Methods for details of the full stochastic cell cycle simulation. The simulation predicts that partitioning by protein amount is required for full size-independence of protein amounts at birth. (C\&D) Protein amount at birth (normalized to the mean) as a function of cell size at birth for WHI5pr-WHI5$m$ Citrine and (C) WHI5pr-WHI5(WIQ)-mCitrine or (D) ACT1pr-mCitrine cells. Data are binned according to cell size at birth and the bin means (+/- SEM) are plotted. Un-binned single-cell values of the same data are plotted in Fig. S6D\&E. 

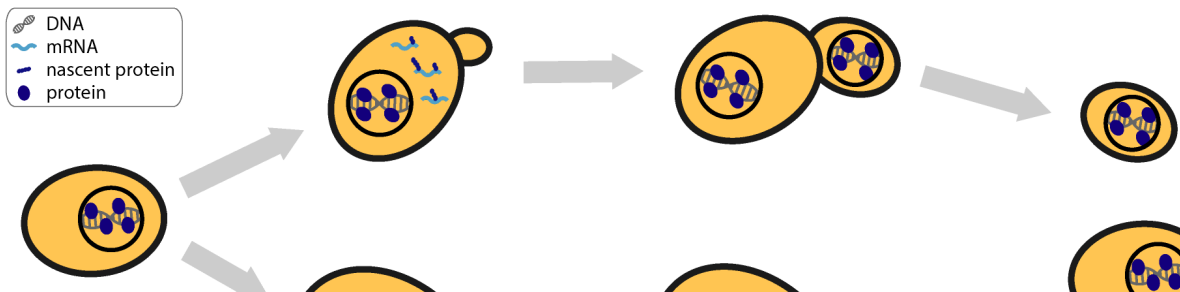

\section{Figure 5 | Summary schematic}

295 A small class of genes including the cell cycle inhibitor WHI5 and histones are transcribed in a sizeindependent manner. This in turn, results in size-independent protein synthesis during the cell cycle.

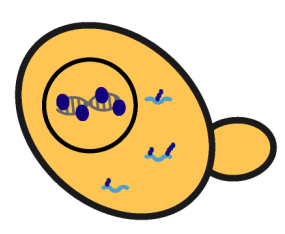

size-independent transcription

\section{size-independent} protein synthesis chromatin-based segregation size-independent inheritance However, alone this is insufficient because, as is the case for most proteins, the asymmetry of cell division results in inheritance of fewer molecules in the smaller daughter cell. Thus, size-independent proteins must also be partitioned during cell division in a size-independent manner, which is achieved through chromatinbinding based partitioning. 
A
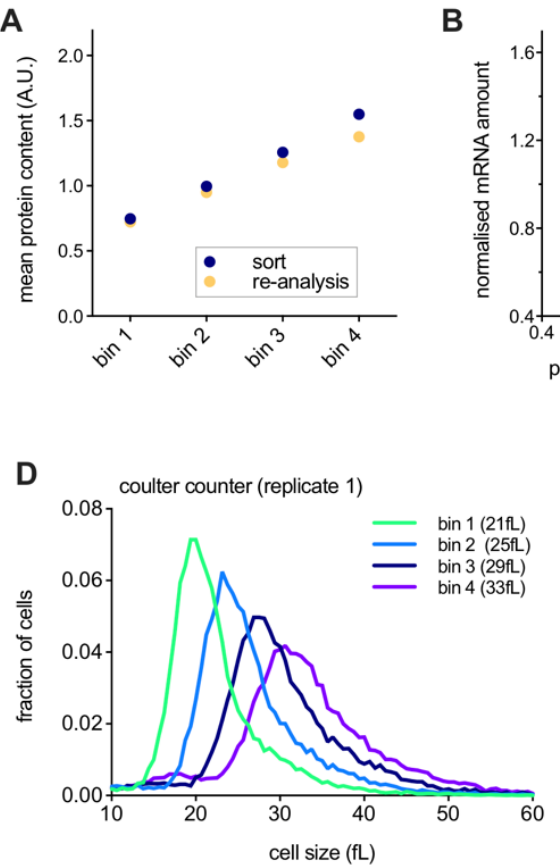

B

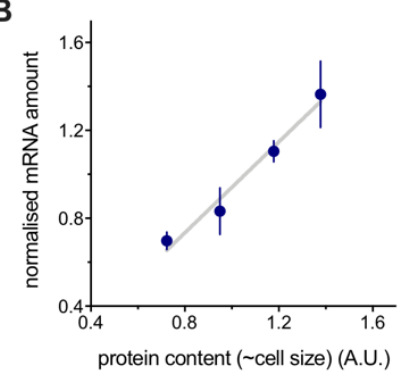

C

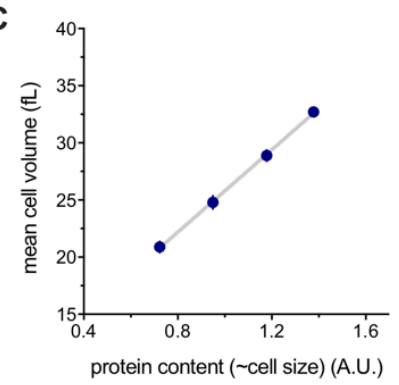

Figure S1 | Transcriptomic analysis of cells sorted by cell size

303 Related to Figures 1D-F.

304 (A-E) Cells in S/G2/M were sorted into four different bins based on the intensity of a total protein dye that 305 we used as a proxy for cell size. See Materials and Methods for further details.

306 (A) Protein dye intensity (normalized to the mean) measured during sorting (blue) and re-measured for cells 307 from each bin after the sort (yellow). The re-analysis (yellow) confirms high sort fidelity.

308 (B) The amount of mRNA (mean +/- SEM) per cell for each bin, determined by the number of reads relative 309 to those from a fixed number of $S$. pombe spike-in cells added to the sample. Total protein amount is well 310 correlated with total cellular mRNA amount.

311 (C-E) Cell volume of cells after sorting, measured by Coulter counter. Total protein amount is correlated 312 with cell volume. 
A

\begin{tabular}{c|c|c} 
cycling & elutriate & $\mathrm{G} 1$ arrest \\
\hline $25^{\circ} \mathrm{C}(\mathrm{SCD})$ & $4^{\circ} \mathrm{C}(\mathrm{SC})$ & $37^{\circ} \mathrm{C}(\mathrm{SCD})$ \\
& $\begin{array}{c}\text { temp shift } \\
\text { tglucose }\end{array}$ & $\begin{array}{l}\text { release }\left(25^{\circ} \mathrm{C}\right) \\
\text { release }\left(25^{\circ} \mathrm{C}\right)\end{array}$ mall \\
release $\left(25^{\circ} \mathrm{C}\right)$ & medium \\
large
\end{tabular}

replicate 1

B

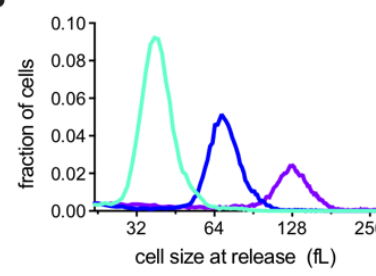

D
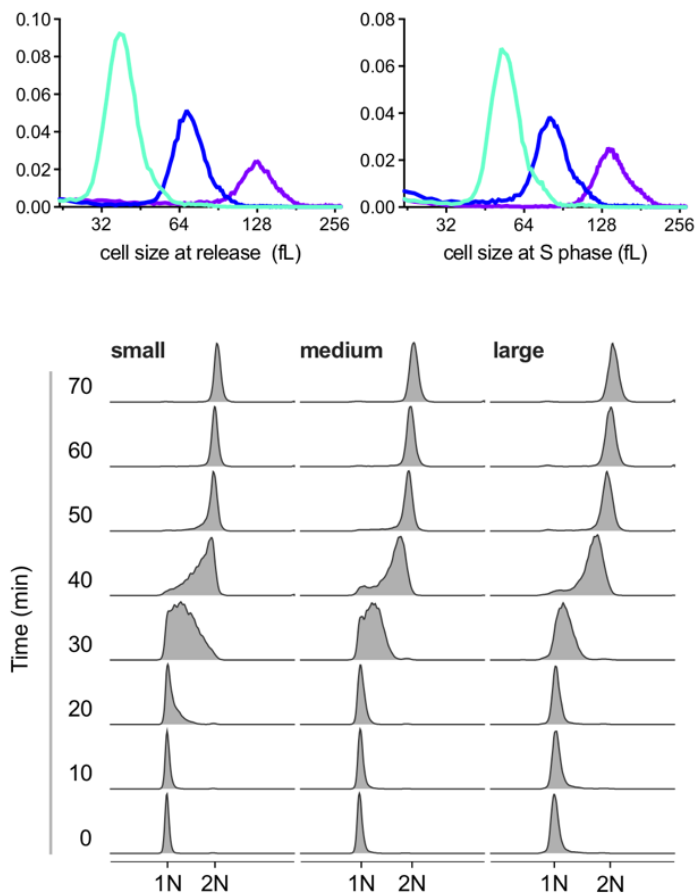

replicate 2

C

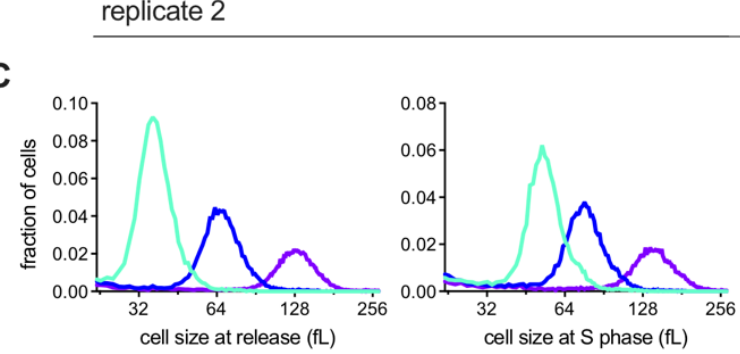

$\mathbf{E}$

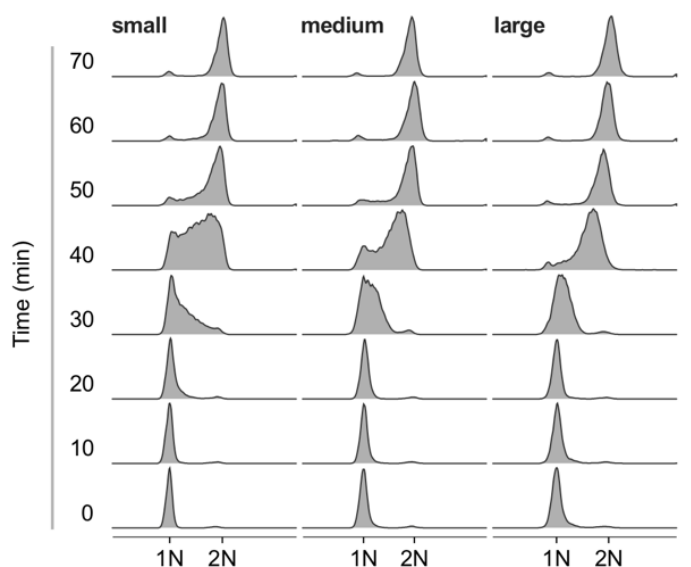

Figure S2 | Transcriptomic analysis of different sized cells synchronously progressing through the cell cycle

Related to Figures $1 \mathrm{~J}-\mathrm{L}$.

(A) Schematic of experimental design. G1 cells harboring a temperature sensitive Cdk1 allele (cdc28-13) were recovered by centrifugal elutriation. Cells were then shifted to the restrictive temperature $\left(37^{\circ} \mathrm{C}\right)$ to arrest them in G1. After increasing amounts of time during the arrest, cells reached small, medium or large cell sizes. The culture was then shifted to the permissive temperature $\left(25^{\circ} \mathrm{C}\right)$ to allow cell cycle entry. Samples for RNA-seq were then collected every ten minutes at the eight time points corresponding to synchronous progression through the cell cycle. The 0 minute time point is designated as that 30 minutes before early $S$ phase as determined by DNA-content analysis using flow cytometry (Fig. S2D\&E). See Materials and Methods for further details.

326 (B\&C) Cell size distributions, measured by Coulter counter, for small, medium, and large cells when they are released from the $\mathrm{G} 1$ arrest (left panel) or at mid S-phase (the 40 min time point, right panel) for two

328 biological replicates. 
bioRxiv preprint doi: https://doi.org/10.1101/2020.08.28.272690; this version posted August 28, 2020. The copyright holder for this preprint (which was not certified by peer review) is the author/funder, who has granted bioRxiv a license to display the preprint in perpetuity. It is made available under aCC-BY 4.0 International license.

329 (D\&E) DNA content analysis of small, medium, and large cells synchronously progressing through the cell 330 cycle for two biological replicates.

331 Note that the data in Figures S2B\&D is also presented in Figure 2A\&B and is shown here for comparison 332 with replicate experiment (S2C\&E). 

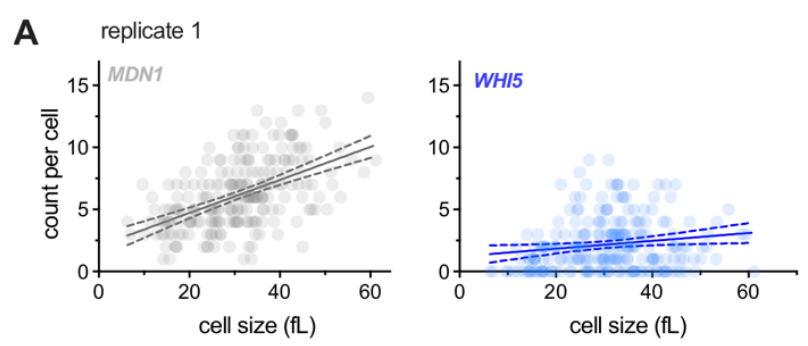

C

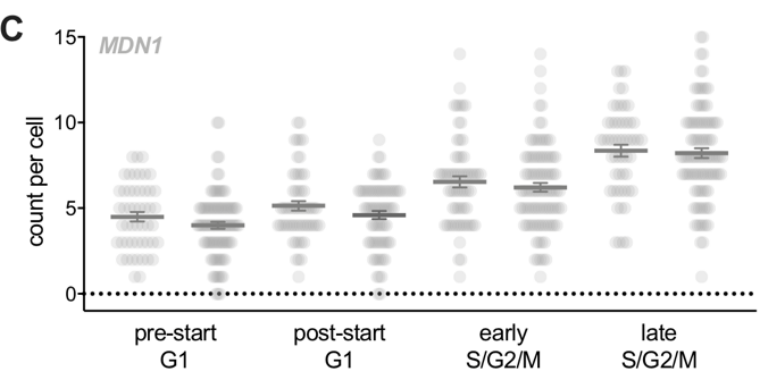

E replicate 1
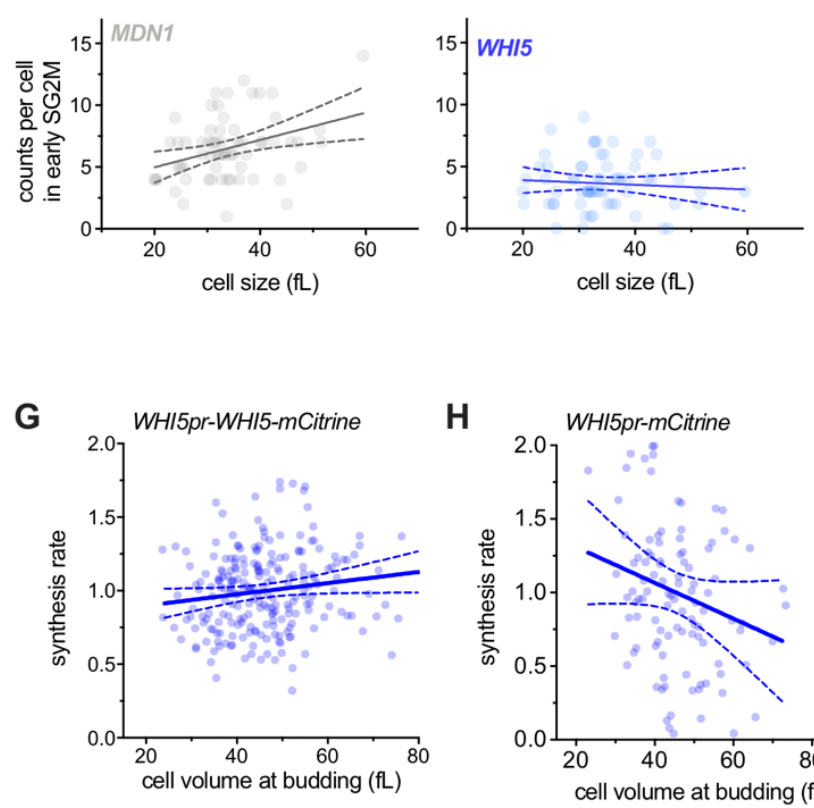

B
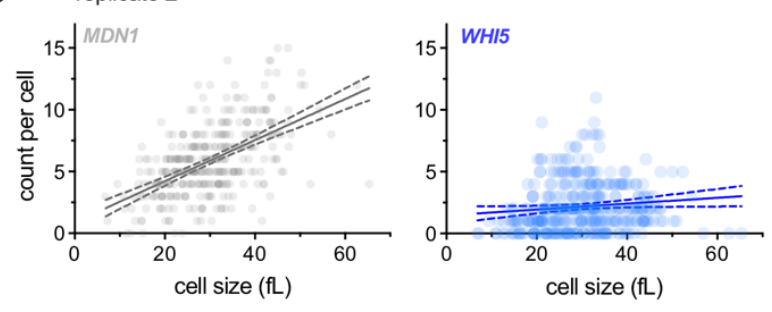

D

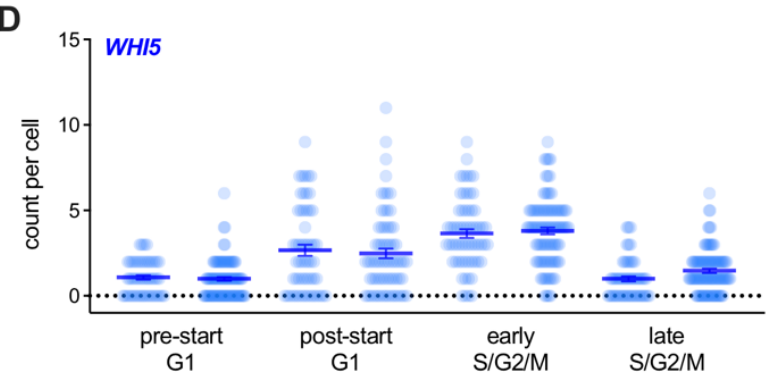

F replicate 2
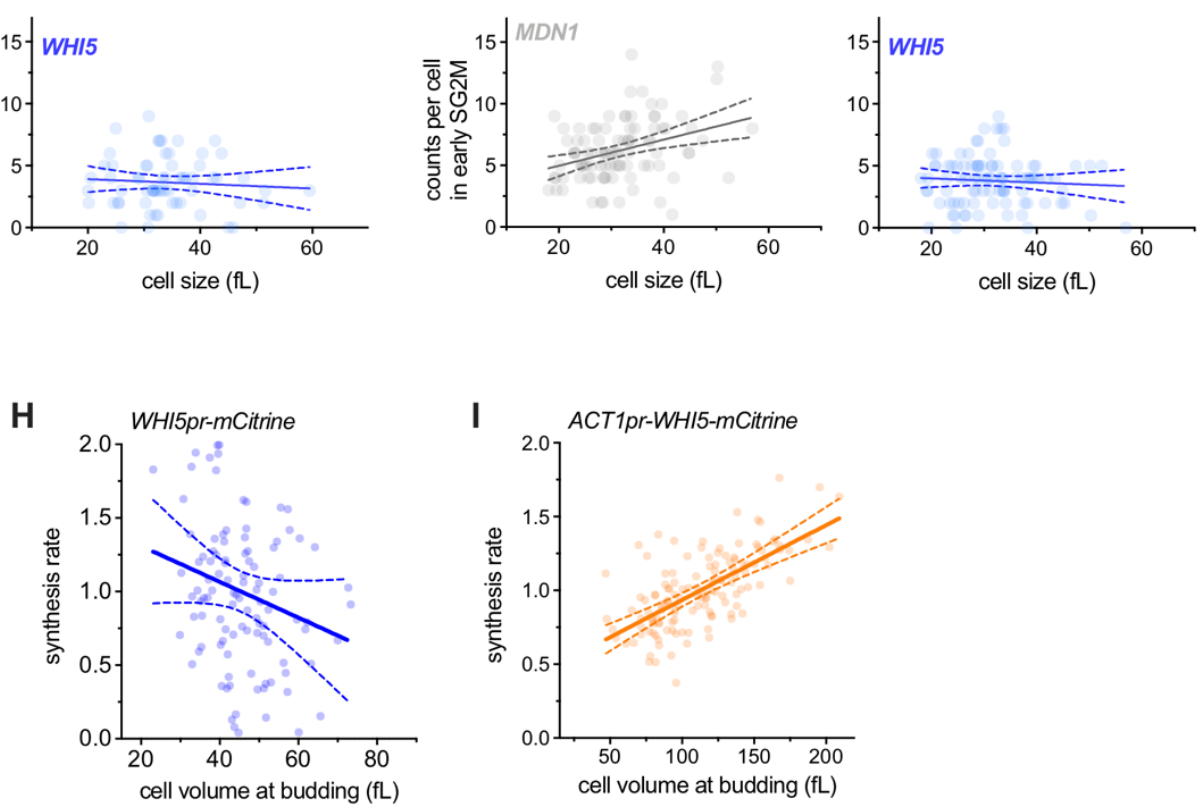

\section{Figure S3 | Single-cell analysis of WHI5 mRNA expression}

Related to Figures 1G-I\&M-P.

(A\&B) mRNA counts per cell, measured by single molecule FISH, for MDN1 or WHI5 as a function of cell size in two independent biological replicates. All cells of any cell cycle stage are included. Linear regression (solid line) and 95\% confidence interval (dashed lines) are shown. The same data are re-plotted in Fig. H\&I with replicates pooled. 
341 (C\&D) mRNA counts per cell, measured by single molecule FISH, for MDN1 or WHI5 at different cell cycle 342 stages in two independent biological replicates. Mean (solid line) +/- SEM (error bars) are shown. See 343 Materials and methods for details of cell cycle stage classification.

344 (E\&F) mRNA counts per cell, measured by single molecule FISH, for MDN1 and WHI5 as a function of cell 345 size during early S/G2/M in two independent biological replicates. Early S/G2/M cells were defined as 346 budded cells with a small ( $\leq 0.2$ ) bud-to-mother volume ratio. Linear regression (solid line) and $95 \%$ 347 confidence interval (dashed lines) are shown. The same data are re-plotted in Fig. 2E with replicates 348 pooled.

349 (G-I) Protein synthesis rates (normalized to the mean) as a function of cell volume at budding, measured 350 by time-lapse microscopy for Whi5-mCitrine fusion proteins expressed from (G) the endogenous WHI5 351 promoter or $(\mathrm{I})$ the $A C T 1$ promoter and $(\mathrm{H})$ mCitrine expressed alone from the WHI5 promoter. Synthesis 352 rates were determined for single cells for the period between bud emergence and cytokinesis using a linear 353 fit as in (Schmoller et al., 2015). Linear regression (solid line) and 95\% confidence interval (dashed lines) 354 are shown. (G) $n=229,2$ data points outside the axis limits. (I) $n=113,4$ data points outside the axis limits. $355(\mathrm{H}) \mathrm{n}=132$. The same data, binned by cell volume at budding, are shown in Fig. 1F-H. 
bioRxiv preprint doi: https://doi.org/10.1101/2020.08.28.272690; this version posted August 28, 2020. The copyright holder for this preprint (which was not certified by peer review) is the author/funder, who has granted bioRxiv a license to display the preprint in perpetuity. It is made available under aCC-BY 4.0 International license.

A

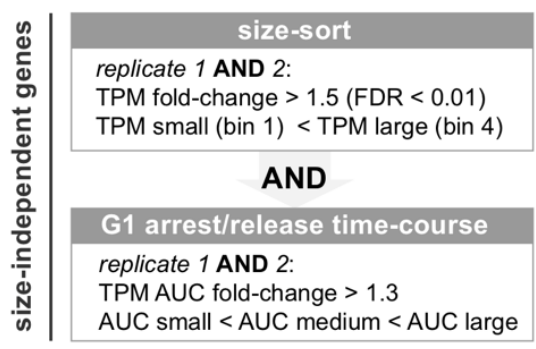

B

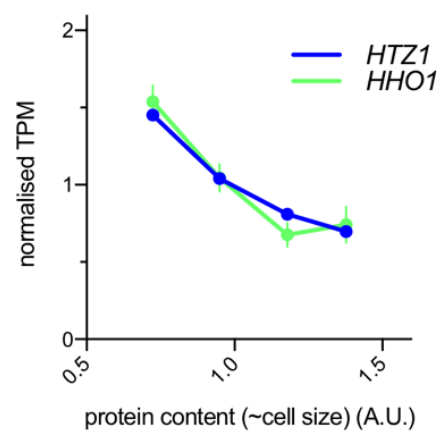

C
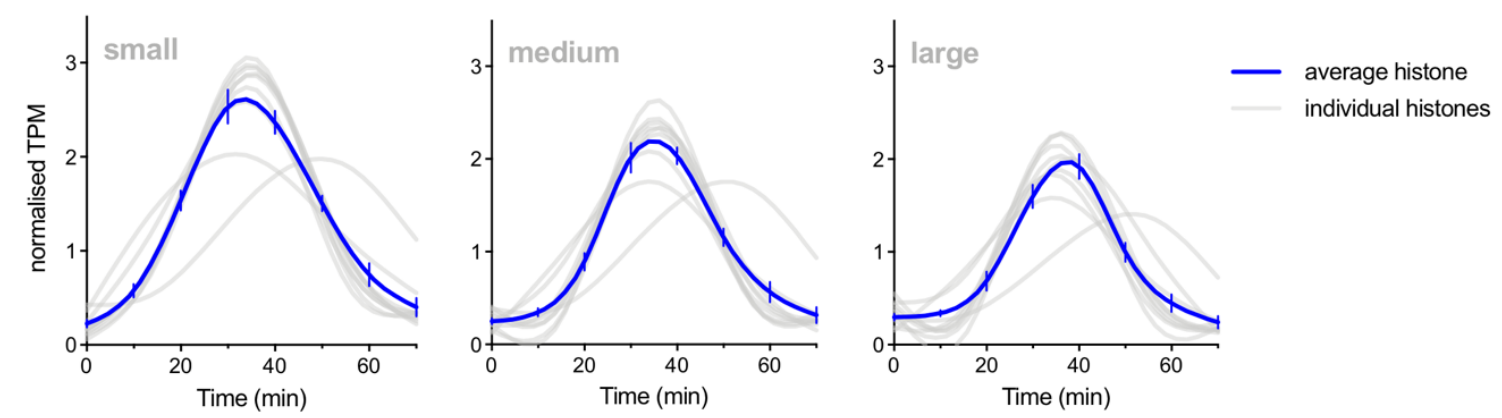

D
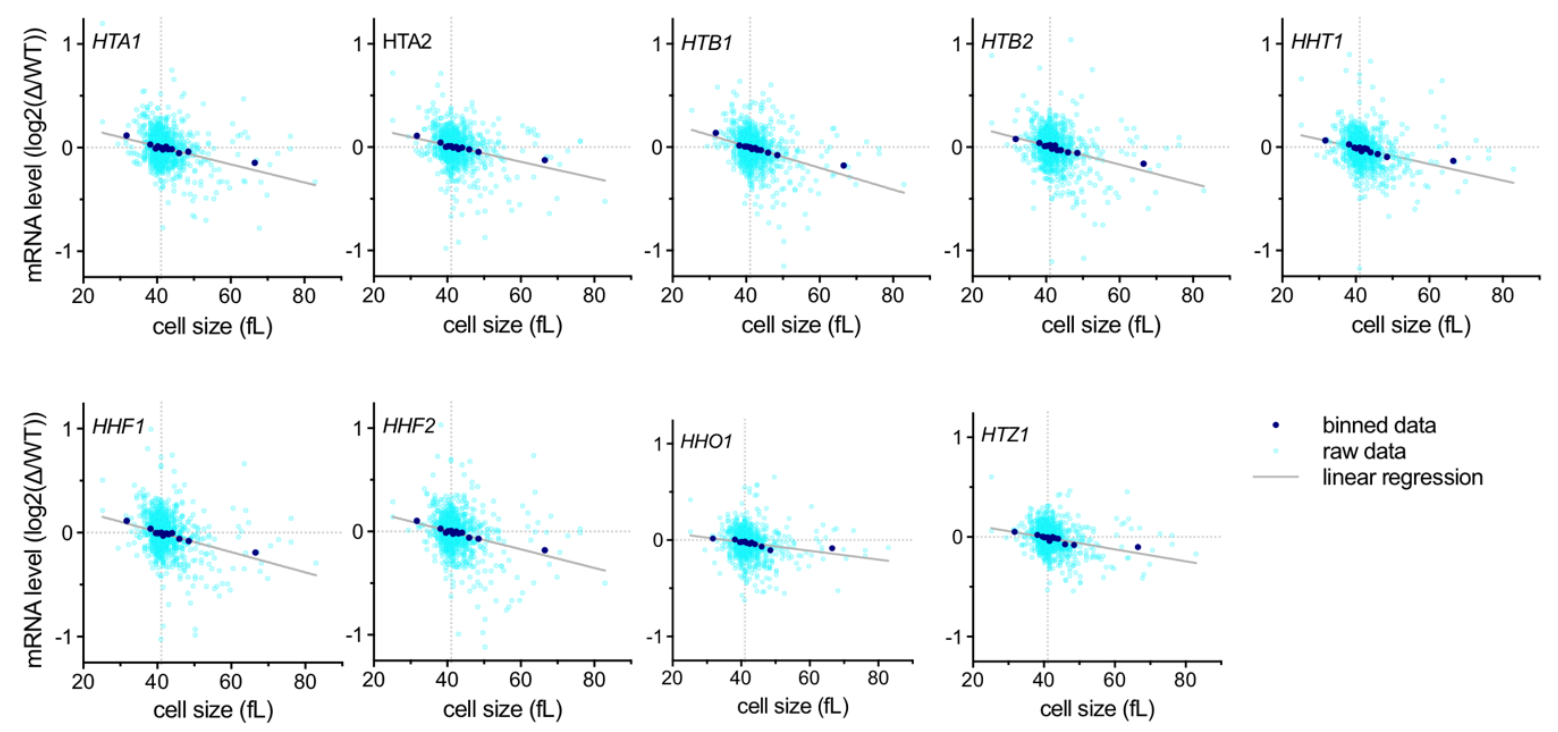

E
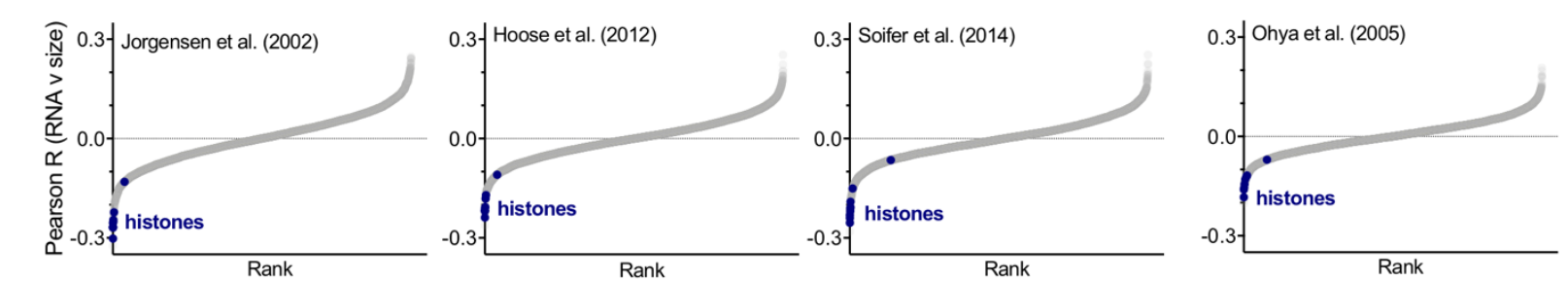
360 (A) Criteria used to classify size-independent genes. Genes whose expression changed in a manner similar 361 to WHI5 in both replicates of the size-sort (see Fig. 1A-C) and in the G1 arrest/release time course (see 362 Fig. 2A-D) were classified as size-independent genes. See Materials and Methods for further details.

363 (B) Normalized TPM (TPM / mean TPM) for HTZ1 and HHO1 mRNA in cells of different sizes (total protein 364 content). Mean (+/- range) of two biological replicates is plotted. Changes in TPM are proportional to 365 changes in mRNA concentration. See Figure S1 and Materials and Methods for further details.

366 (C) Normalized histone mRNA TPM (TPM / mean TPM) of small, medium or large cells at 10-minute time 367 intervals synchronously progressing through the cell cycle. A smoothing spline fitted to the mean normalized 368 TPM from two biological replicates for each histone is plotted in grey. A Spline fitted to mean (+/- SEM) of 369 all histones is shown in blue.

370 (D) Comparison of histone mRNA levels in 1,484 gene deletions, relative to wild-type (Kemmeren et al., 371 2014; O'Duibhir et al., 2014) with cell size of the respective gene deletions from Jorgensen et al., 2002.

372 See Materials and Methods for details. 
A
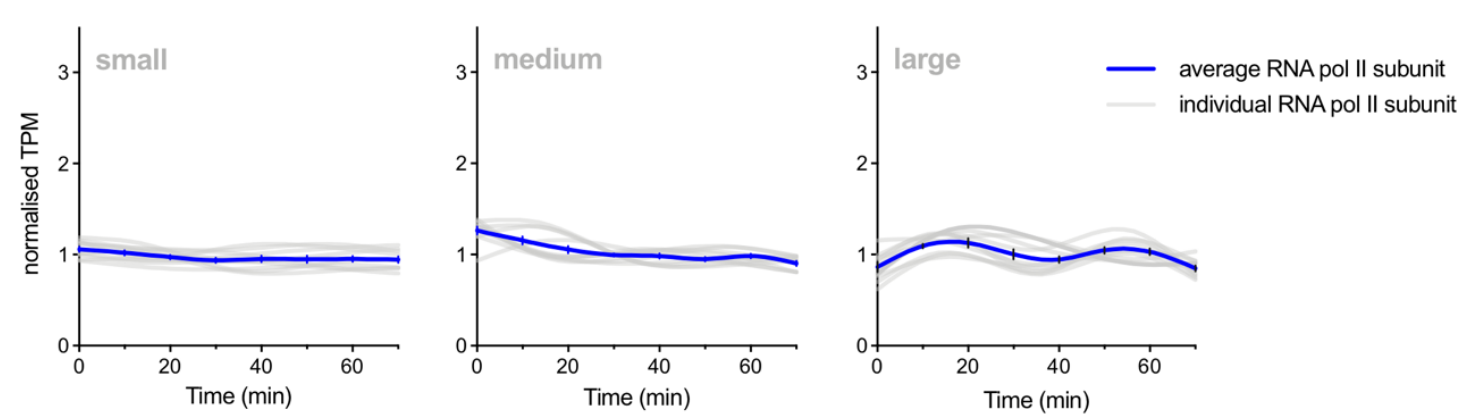

B

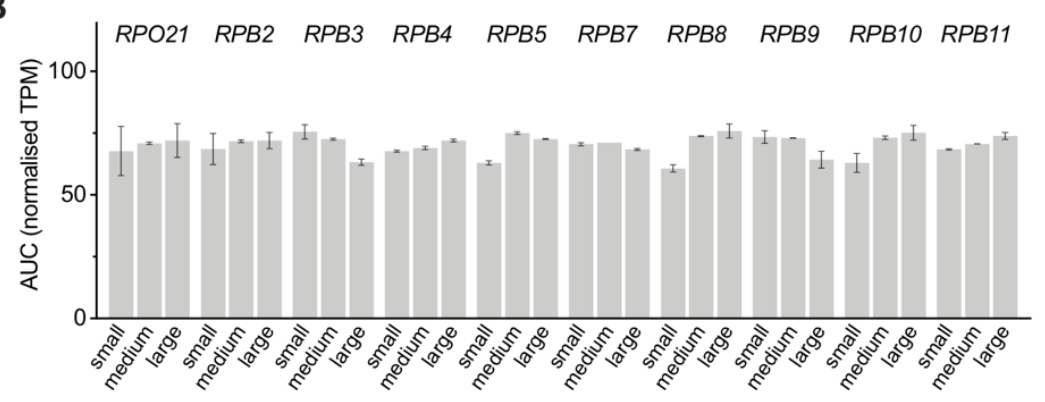

C
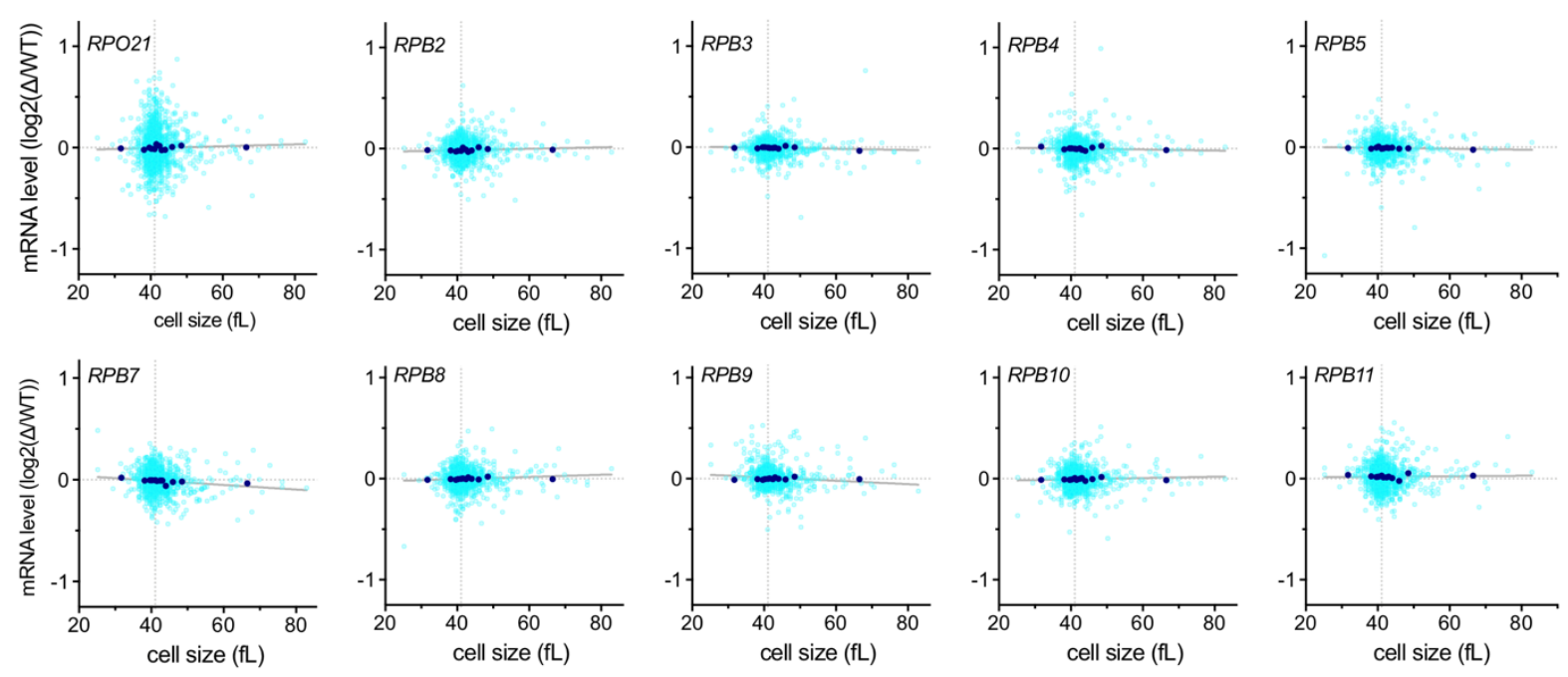

- binned data

raw data

linear regression

D
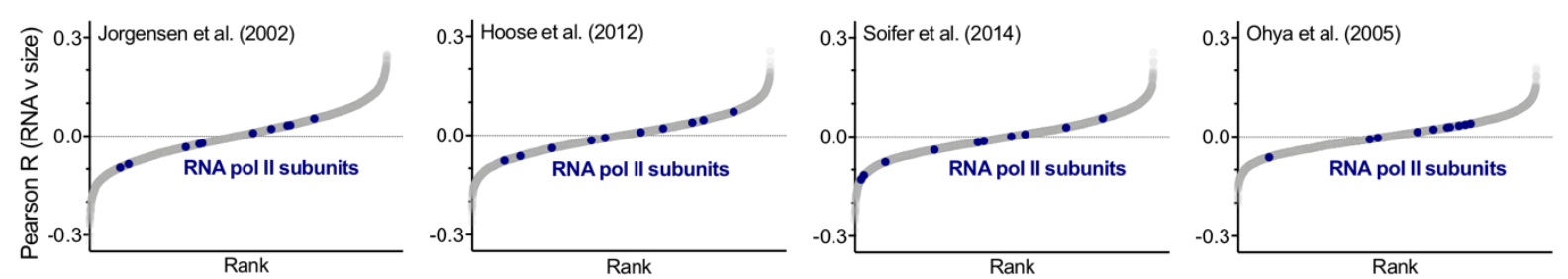
378 to the average normalized TPM from two biological replicates for each subunit is plotted in grey. A Spline 379 fitted to the mean (+/- SEM) of all subunits is shown in blue.

380 (B) The Area Under the Curve (AUC) of mean normalized RNA polymerase Il subunit mRNA TPM of small, 381 medium, and large cells synchronously progressing through the cell cycle. The AUC mean (+/- range) of 382 two biological replicates is plotted. 
A

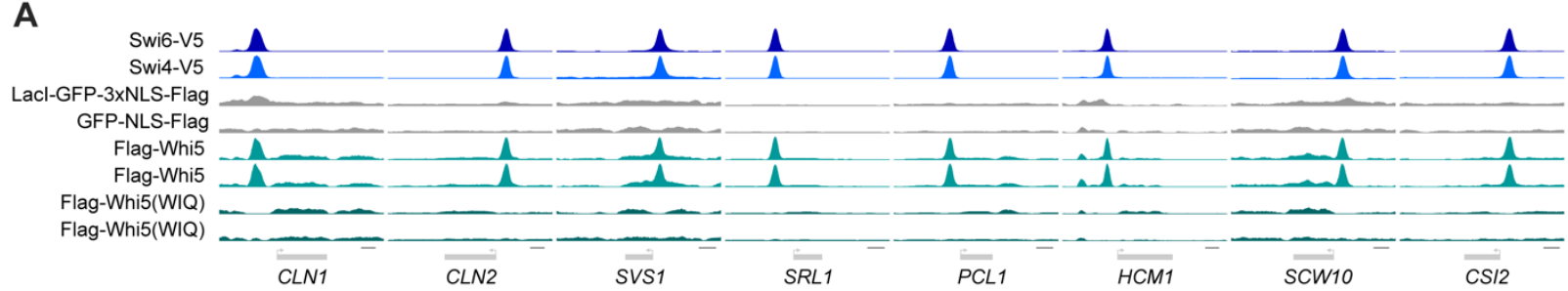

B
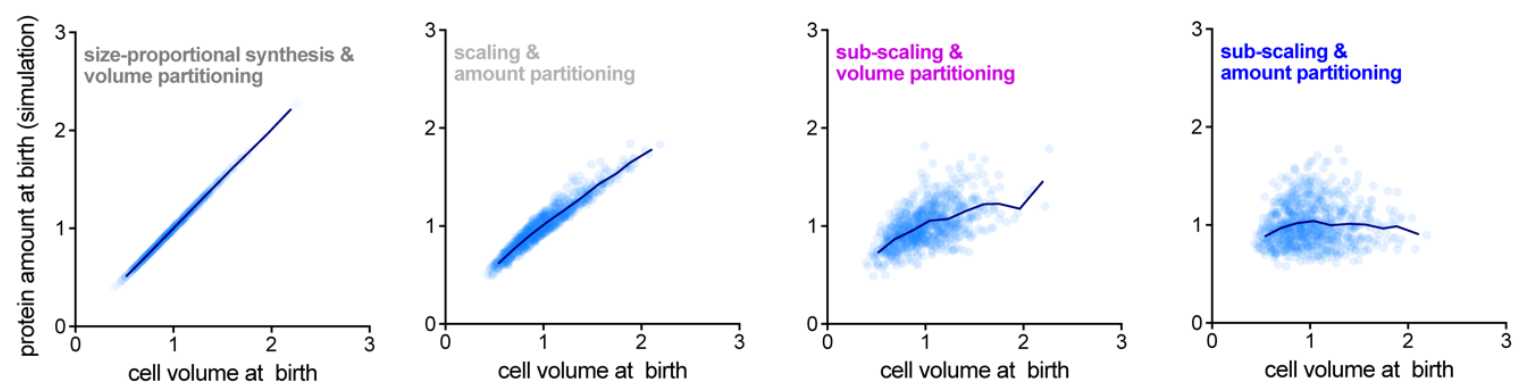

C
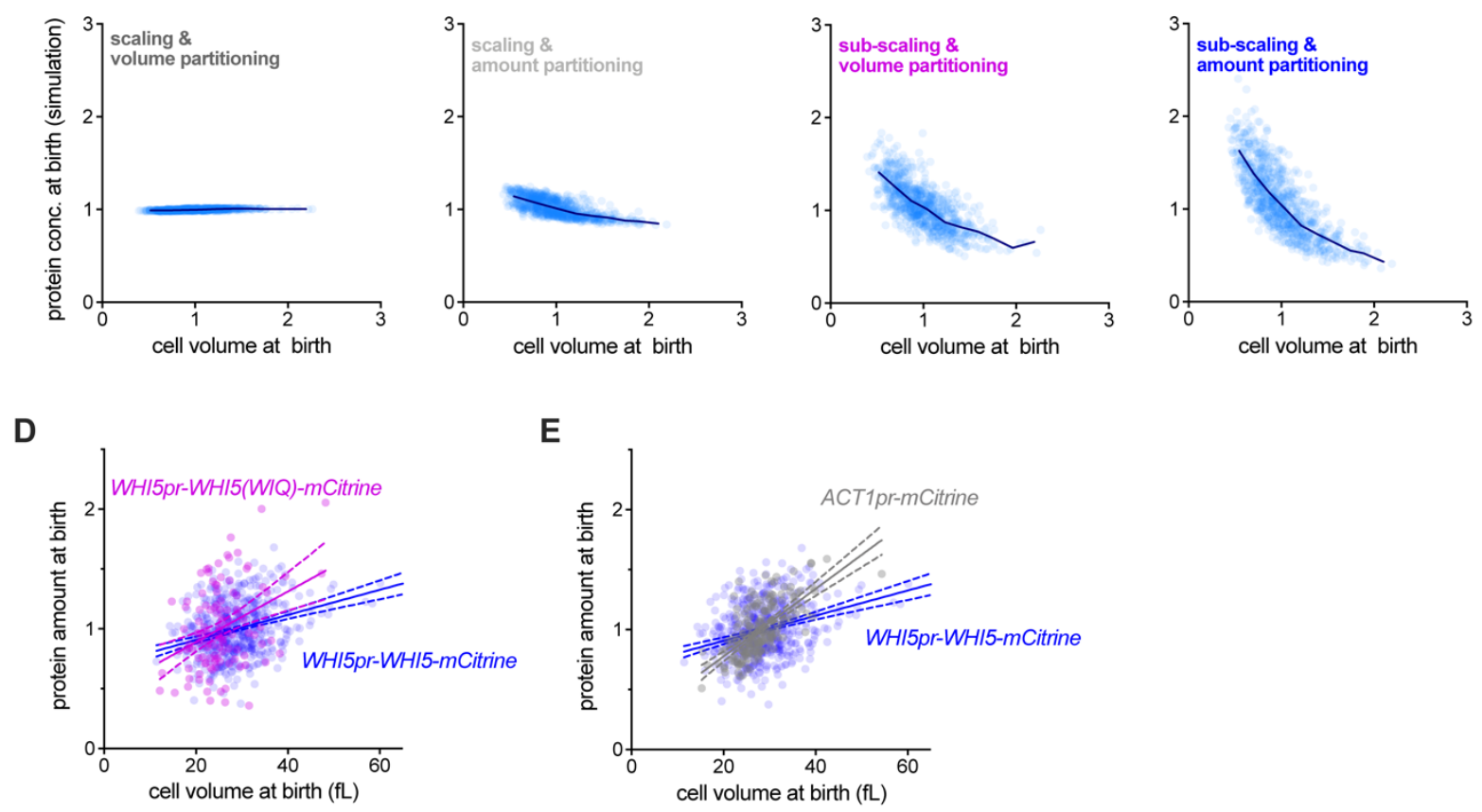

Figure S6 | Size-independent expression is inherited through asymmetric division due to DNAmediated partitioning

Related to Figure 3. (A) anti-V5 or anti-Flag ChIP-seq experiments were performed using cells expressing the indicated fusion proteins (left hand side). Data are shown for 8 example SBF binding sites near the denoted genes. Cells expressing Lacl-GFP-3xNLS-FLAG or GFP-NLS-FLAG were included as controls for non-specific ChIP 
392 signal. Whi5 recruitment to DNA overlaps with SBF (Swi4 and Swi6) binding sites but is lost in the 393 Whi5(WIQ) variant (Travesa et al., 2013).

394 (B\&C) Simulation of (B) protein amount or (C) protein concentration at birth as a function of daughter volume 395 at birth. Four different conditions were simulated where protein expression was either in proportion to cell 396 size or independent of cell size, and protein partitioning is either by amount or in proportion to cell volume. 397 Individual simulated cells (light blue) as well as bin means (dark blue) are plotted. Note binned values for 398 (B) are also plotted in Figure 3B.

399 (D\&E) Protein amount at birth for WHI5pr-WHI5-mCitrine and (D) WHI5pr-WHI5(WIQ)-mCitrine or (E) 400 ACT1pr-mCitrine cells. Note, Fig. 4C\&D shows bin means of the same data binned by cell volume at birth. 
401 Table S1 - list of S. cerevisiae strain used in this study and full genotypes

\begin{tabular}{|c|c|c|}
\hline A17896 & Amon lab & W303; MATa, ADE2, GAL, cdc28-13 \\
\hline DCB99 & This study & W303; MATa, ADE2, WHI5-mCitrine::URA3 \\
\hline MK551-1 & This study & W303; MATa, bar1 $\Delta$, HISG, whi5 $\Delta:: L E U 2, \operatorname{cln} 3 \Delta:: H p h M X$ \\
\hline HTA1-GFP & $\begin{array}{l}\text { GFP collection } \\
\text { (Huh et al., 2003) }\end{array}$ & BY4741; HTA1-GFP \\
\hline HTB2-GFP & $\begin{array}{l}\text { GFP collection } \\
\text { (Huh et al., 2003) }\end{array}$ & BY4741; HTB2-GFP \\
\hline HTZ1-GFP & $\begin{array}{l}\text { GFP collection } \\
\text { (Huh et al., 2003) }\end{array}$ & BY4741; HTZ1-GFP \\
\hline RPB3-GFP & $\begin{array}{l}\text { GFP collection } \\
\text { (Huh et al., 2003) }\end{array}$ & BY4741; RPB3-GFP \\
\hline DCB72 & This study & W303; MATa, ADE2, ura3:: WHI5pr(1kb)-mCitrine-CYC1term \\
\hline KSY108-1 & Lab collection & W303; ADE2, WHI5-mCitrine-HIS3 \\
\hline KSY190-2 & This study & $\begin{array}{l}\text { W303; ADE2, whi5s::KanMX URA3::WHI5pr(1kb)-WHI5-WIQ-mCitrine- } \\
\text { ADH1term }\end{array}$ \\
\hline KSY160-2 & This study & W303; ADE, URA3::ACT1pr(1kb) -Whi5-mCitrine-CYC1term \\
\hline KSY158-1 & Lab collection & W303; ADE2, URA3:: ACT1pr(1kb)-mCitrine-CYC1term \\
\hline MS534 & This study & W303; bar1A::HisG GFP-3xNLS-FLAG \\
\hline MS535 & This study & W303; bar1 ::HisG Lacl-GFP-3xNLS-FLAG \\
\hline MS536 & This study & 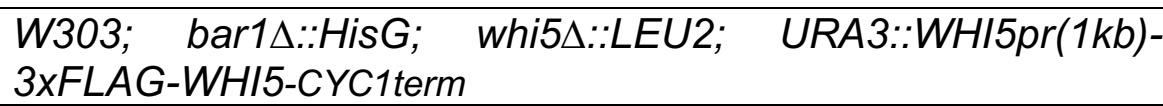 \\
\hline MS537 & This study & $\begin{array}{l}\text { W303; bar1 } \quad: \text { HisG; } \quad \text { URA3::WHI5pr(1kb)-3xFLAG- } \\
\text { WHI5(WIQ)-CYC1term }\end{array}$ \\
\hline MK653-1 & This study & W303; bar14; SWI4-V5::hphMX6 \\
\hline MK645-1 & This study & W303; bar1A; SWI6-V5::hphMX6 \\
\hline
\end{tabular}


Chandler-Brown, D., Schmoller, K.M., Winetraub, Y., and Skotheim, J.M. (2017). The Adder Phenomenon Emerges from Independent Control of Pre- and Post-Start Phases of the Budding Yeast Cell Cycle. Curr Biol 27, 2774-2783 e2773. Chen, Y., Zhao, G., Zahumensky, J., Honey, S., and Futcher, B. (2020). Differential Scaling of Gene Expression with Cell Size May Explain Size Control in Budding Yeast. Mol Cell 78, 359-370 e356. Chia, W., Somers, W.G., and Wang, H. (2008). Drosophila neuroblast asymmetric divisions: cell cycle regulators, asymmetric protein localization, and tumorigenesis. J Cell Biol 180, 267-272. Creanor, J., and Mitchison, J.M. (1982). Patterns of protein synthesis during the cell cycle of the fission yeast Schizosaccharomyces pombe. J Cell Sci 58, 263-285.

Cross, S.L., and Smith, M.M. (1988). Comparison of the structure and cell cycle expression of mRNAs encoded by two histone H3-H4 loci in Saccharomyces cerevisiae. Mol Cell Biol 8, 945-954. Dobin, A., Davis, C.A., Schlesinger, F., Drenkow, J., Zaleski, C., Jha, S., Batut, P., Chaisson, M., and Gingeras, T.R. (2013). STAR: ultrafast universal RNA-seq aligner. Bioinformatics 29, 15-21. Doncic, A., Eser, U., Atay, O., and Skotheim, J.M. (2013). An algorithm to automate yeast segmentation and tracking. PLoS One 8, e57970.

Doncic, A., Falleur-Fettig, M., and Skotheim, J.M. (2011). Distinct interactions select and maintain a specific cell fate. Mol Cell 43, 528-539.

Eden, E., Navon, R., Steinfeld, I., Lipson, D., and Yakhini, Z. (2009). GOrilla: a tool for discovery and visualization of enriched $\mathrm{GO}$ terms in ranked gene lists. BMC Bioinformatics 10, 48.

Elliott, S.G. (1983). Coordination of growth with cell division: regulation of synthesis of RNA during the cell cycle of the fission yeast Schizosaccharomyces pombe. Mol Gen Genet 192, 204-211. Elliott, S.G., and McLaughlin, C.S. (1979). Regulation of RNA synthesis in yeast. III. Synthesis during the cell cycle. Mol Gen Genet 169, 237-243.

Elliott, S.G., Warner, J.R., and McLaughlin, C.S. (1979). Synthesis of ribosomal proteins during the cell cycle of the yeast Saccharomyces cerevisiae. J Bacteriol 137, 1048-1050.

Feng, J., Liu, T., Qin, B., Zhang, Y., and Liu, X.S. (2012). Identifying ChIP-seq enrichment using MACS. Nat Protoc 7, 1728-1740.

Fraser, R.S., and Nurse, P. (1978). Novel cell cycle control of RNA synthesis in yeast. Nature 271, 726-730.

Fraser, R.S., and Nurse, P. (1979). Altered patterns of ribonucleic acid synthesis during the cell cycle: a mechanism compensating for variation in gene concentration. J Cell Sci 35, 25-40. Gunjan, A., and Verreault, A. (2003). A Rad53 kinase-dependent surveillance mechanism that regulates histone protein levels in S. cerevisiae. Cell 115, 537-549.

Hoose, S.A., Rawlings, J.A., Kelly, M.M., Leitch, M.C., Ababneh, Q.O., Robles, J.P., Taylor, D., Hoover, E.M., Hailu, B., McEnery, K.A., et al. (2012). A systematic analysis of cell cycle regulators in yeast reveals that most factors act independently of cell size to control initiation of division. PLoS Genet 8, e1002590.

Hu, B., Petela, N., Kurze, A., Chan, K.L., Chapard, C., and Nasmyth, K. (2015). Biological chromodynamics: a general method for measuring protein occupancy across the genome by calibrating ChIP-seq. Nucleic Acids Res 43, e132.

Huh, W.K., Falvo, J.V., Gerke, L.C., Carroll, A.S., Howson, R.W., Weissman, J.S., and O'Shea, E.K. (2003). Global analysis of protein localization in budding yeast. Nature 425, 686-691. Jorgensen, P., Edgington, N.P., Schneider, B.L., Rupes, I., Tyers, M., and Futcher, B. (2007). The size of the nucleus increases as yeast cells grow. Mol Biol Cell 18, 3523-3532. Jorgensen, P., Nishikawa, J.L., Breitkreutz, B.J., and Tyers, M. (2002). Systematic identification of pathways that couple cell growth and division in yeast. Science 297, 395-400.

Keifenheim, D., Sun, X.M., D'Souza, E., Ohira, M.J., Magner, M., Mayhew, M.B., Marguerat, S., and Rhind, N. (2017). Size-Dependent Expression of the Mitotic Activator Cdc25 Suggests a Mechanism 
Kemmeren, P., Sameith, K., van de Pasch, L.A., Benschop, J.J., Lenstra, T.L., Margaritis, T., O'Duibhir, E., Apweiler, E., van Wageningen, S., Ko, C.W., et al. (2014). Large-scale genetic perturbations reveal regulatory networks and an abundance of gene-specific repressors. Cell 157, 740-752. Langmead, B., Trapnell, C., Pop, M., and Salzberg, S.L. (2009). Ultrafast and memory-efficient alignment of short DNA sequences to the human genome. Genome Biol 10, R25. Lock, A., Rutherford, K., Harris, M.A., Hayles, J., Oliver, S.G., Bahler, J., and Wood, V. (2019). PomBase 2018: user-driven reimplementation of the fission yeast database provides rapid and intuitive access to diverse, interconnected information. Nucleic Acids Res 47, D821-D827. Love, M.I., Huber, W., and Anders, S. (2014). Moderated estimation of fold change and dispersion for RNA-seq data with DESeq2. Genome Biol 15, 550.

Marguerat, S., and Bahler, J. (2012). Coordinating genome expression with cell size. Trends Genet 28, 560-565.

Marguerat, S., Schmidt, A., Codlin, S., Chen, W., Aebersold, R., and Bahler, J. (2012). Quantitative analysis of fission yeast transcriptomes and proteomes in proliferating and quiescent cells. Cell 151, 671-683.

Moran, L., Norris, D., and Osley, M.A. (1990). A yeast H2A-H2B promoter can be regulated by changes in histone gene copy number. Genes Dev 4, 752-763.

Neumann, F.R., and Nurse, P. (2007). Nuclear size control in fission yeast. J Cell Biol 179, 593-600. Neurohr, G.E., Terry, R.L., Lengefeld, J., Bonney, M., Brittingham, G.P., Moretto, F., Miettinen, T.P., Vaites, L.P., Soares, L.M., Paulo, J.A., et al. (2019). Excessive Cell Growth Causes Cytoplasm Dilution And Contributes to Senescence. Cell 176, 1083-1097 e1018. Norris, D., and Osley, M.A. (1987). The two gene pairs encoding H2A and H2B play different roles in the Saccharomyces cerevisiae life cycle. Mol Cell Biol 7, 3473-3481. O'Duibhir, E., Lijnzaad, P., Benschop, J.J., Lenstra, T.L., van Leenen, D., Groot Koerkamp, M.J., Margaritis, T., Brok, M.O., Kemmeren, P., and Holstege, F.C. (2014). Cell cycle population effects in perturbation studies. Mol Syst Biol 10, 732.

Ohya, Y., Sese, J., Yukawa, M., Sano, F., Nakatani, Y., Saito, T.L., Saka, A., Fukuda, T., Ishihara, S., Oka, S., et al. (2005). High-dimensional and large-scale phenotyping of yeast mutants. Proc Natl Acad Sci U S A 102, 19015-19020.

Padovan-Merhar, O., Nair, G.P., Biaesch, A.G., Mayer, A., Scarfone, S., Foley, S.W., Wu, A.R., Churchman, L.S., Singh, A., and Raj, A. (2015). Single mammalian cells compensate for differences in cellular volume and DNA copy number through independent global transcriptional mechanisms. Mol Cell 58, 339-352.

Parts, L., Liu, Y.C., Tekkedil, M.M., Steinmetz, L.M., Caudy, A.A., Fraser, A.G., Boone, C., Andrews, B.J., and Rosebrock, A.P. (2014). Heritability and genetic basis of protein level variation in an outbred population. Genome Res 24, 1363-1370. Pramila, T., Wu, W., Miles, S., Noble, W.S., and Breeden, L.L. (2006). The Forkhead transcription factor $\mathrm{Hcm} 1$ regulates chromosome segregation genes and fills the S-phase gap in the transcriptional circuitry of the cell cycle. Genes Dev 20, 2266-2278. Qu, Y., Jiang, J., Liu, X., Wei, P., Yang, X., and Tang, C. (2019). Cell Cycle Inhibitor Whi5 Records Environmental Information to Coordinate Growth and Division in Yeast. Cell Rep 29, 987-994 e985. Raj, A., and Tyagi, S. (2010). Detection of individual endogenous RNA transcripts in situ using multiple singly labeled probes. Methods Enzymol 472, 365-386.

Roberts, A., and Pachter, L. (2013). Streaming fragment assignment for real-time analysis of sequencing experiments. Nat Methods 10, 71-73.

Schmoller, K.M., Turner, J.J., Koivomagi, M., and Skotheim, J.M. (2015). Dilution of the cell cycle inhibitor Whi5 controls budding-yeast cell size. Nature 526, 268-272.

Shipony, Z., Marinov, G.K., Swaffer, M.P., Sinnott-Armstrong, N.A., Skotheim, J.M., Kundaje, A., and Greenleaf, W.J. (2020). Long-range single-molecule mapping of chromatin accessibility in eukaryotes. Nat Methods.

506 Syst Biol 10, 761. 
Sulston, J.E., Schierenberg, E., White, J.G., and Thomson, J.N. (1983). The embryonic cell lineage of the nematode Caenorhabditis elegans. Dev Biol 100, 64-119. Sun, X.M., Bowman, A., Priestman, M., Bertaux, F., Martinez-Segura, A., Tang, W., Whilding, C., Dormann, D., Shahrezaei, V., and Marguerat, S. (2020). Size-Dependent Increase in RNA Polymerase II Initiation Rates Mediates Gene Expression Scaling with Cell Size. Curr Biol 30, 1217-

513 Travesa, A., Kalashnikova, T.I., de Bruin, R.A., Cass, S.R., Chahwan, C., Lee, D.E., Lowndes, N.F., 514 and Wittenberg, C. (2013). Repression of G1/S transcription is mediated via interaction of the GTB 515 motifs of Nrm1 and Whi5 with Swi6. Mol Cell Biol 33, 1476-1486.

516 Trcek, T., Chao, J.A., Larson, D.R., Park, H.Y., Zenklusen, D., Shenoy, S.M., and Singer, R.H.

517 (2012). Single-mRNA counting using fluorescent in situ hybridization in budding yeast. Nat Protoc 7 , 518 408-419.

519 Tutucci, E., Vera, M., Biswas, J., Garcia, J., Parker, R., and Singer, R.H. (2018). An improved MS2 system for accurate reporting of the mRNA life cycle. Nat Methods 15, 81-89. Youk, H., Raj, A., and van Oudenaarden, A. (2010). Imaging single mRNA molecules in yeast. Methods Enzymol 470, 429-446. Zatulovskiy, E., Zhang, S., Berenson, D.F., Topacio, B.R., and Skotheim, J.M. (2020). Cell growth dilutes the cell cycle inhibitor $\mathrm{Rb}$ to trigger cell division. Science 369, 466-471. Zenklusen, D., Larson, D.R., and Singer, R.H. (2008). Single-RNA counting reveals alternative modes of gene expression in yeast. Nat Struct Mol Biol 15, 1263-1271. global control over cellular transcription. Curr Biol 20, 2010-2015. 
530 Acknowledgments. We would like to thank Bruce Futcher and Yuping Chen for invaluable advice on

531 centrifugal elutriation, members of the Skotheim laboratory for constructive feedback, Gabriel Neurohr

532 for sending strain A17896, Chris You for assistance with the ChIP-seq experiments and Jon Turner

533 for help in optimizing the smFISH protocol. This work was supported by the NIH (GM092925 and

534 GM115479), the HHMI-Simons (JMS, Faculty Scholars Program). MPS was supported by a Simons

535 Foundation Fellowship of the Life Sciences Research Foundation and an EMBO Long-Term

536 Postdoctoral Fellowship. KMS was supported by the Human Frontier Science Program (Postdoctoral

537 Fellowship and Career Development Award).

538

539 Author contributions. JMS supervised the work. MPS and JMS wrote the manuscript. MPS

540 performed and analyzed all experiments except for live cell microscopy experiments performed and

541 analyzed by DCB \& KMS and smFISH experiments performed and analyzed by ML. Sequencing data

542 analysis was performed by MPS and GM, supervised by WG \& AK.

543

544 Conflict of interests. The authors declare no conflicts of interest. 


\section{MATERIALS AND METHODS}

\section{Yeast genetics}

548 Standard procedures were used for Saccharomyces cerevisiae strain construction. Full genotypes of all strains used in this study are listed in Table S1.

Transcriptomic analysis of different sized cells synchronously progressing through the cell 552 cycle

553 To determine transcript levels in cells of different sizes, S/G2/M cells were sorted according to total 554 protein content by fluorescence activated cell sorting (FACS) before RNA extraction and sequencing. $500 \mathrm{ml} \mathrm{S}$. cerevisiae (HTB2-GFP) was grown (synthetic complete media $+2 \%$ glucose at $30^{\circ} \mathrm{C}$ ), and fixed at O.D. $~ 0.3$ by addition of $500 \mathrm{ml} 80 \%$ methanol $20 \mathrm{mM}$ TRIS $\left(-20^{\circ} \mathrm{C}\right)$ and then incubated at $20^{\circ} \mathrm{C}$ for 30 minutes. Cells were fixed to prevent gene expression changes during the course of the cell sorting, which requires multiple generations equivalents of time to complete. Cells were pelleted (13krpm, 3 minutes) and washed $3 x$ in PBS, before gentle sonication and then addition of $5 \mu \mathrm{g} / \mathrm{ml}$ total protein dye (Alexa Fluor ${ }^{\mathrm{TM}} 647$ NHS Ester dye; ThermoFisher Scientific; A20006) and incubation $\left(4^{\circ} \mathrm{C}\right.$, 30 minutes). Cells were again pelleted (13krpm, 3 minutes) and washed $3 x$ in PBS to remove excess dye before again being sonicated. Cells from four different size fractions were sorted on a FACSAria II sorter (BD Biosciences) according to the following strategy. First singlets were gated based on scatter (FSC and SSC), then S/G2/M cells were identified using an Htb2-GFP signal, and then finally, four bins of different total protein content cells were sorted based on the total protein intensity (bin 1 = lowest signal, bin 4 = highest, Fig. S1A). The fidelity of the total protein dye sort was confirmed by reanalyzing 10,000 cells on the same sorter (Fig. 1D \& S1A). Furthermore, total protein content was validated as a proxy for size by measuring the cell volume of the different protein dye sorted cells using a Coulter counter (Fig. S1C-E).

Two biological replicates were performed. Within each biological replicate, two technical replicates were performed for each size bin so that four replicates were performed per bin in total. For

572 the different bins within the same replicate set, a constant number of S. pombe cells, fixed as above,

573 were added as a spike-in to measure total RNA content per $S$. cerevisiae cell. The number of $S$.

574 cerevisiae cells and S. pombe cells (972 h-) per sample was constant within a set of replicates but 575 varied slightly between each set of replicates (5-10 million $S$. cerevisiae cells and 5-10 million $S$. 576 pombe cells). To maximize the number of reads from the experimental $S$. cerevisiae samples, $S$. 577 pombe cells were nitrogen starved (grown in EMM before media switch to EMM $-\mathrm{NH}_{4} \mathrm{Cl}$ for $24 \mathrm{hr}$ at $30^{\circ} \mathrm{C}$ ) because this reduces their mRNA copy number per cell (and their cell size) (Marguerat et al., 2012). Cells were then pelleted (4krpm, 15 minutes), and their RNA extracted and sequenced as 
described below. For each set of replicates, the S. pombe spike-in was added independently and RNA was extracted independently.

To estimate the relative amount of mRNA per cell in each size bin, the number of $S$. cerevisiae reads per S. pombe read was calculated (see RNA-seq data processing below) and then normalized to the mean value within a set of replicates. The normalized total mRNA per sample was then averaged between the four replicates (Fig. S1B).

Transcriptomic analysis of different sized cells synchronously progressing through the cell cycle

To determine transcript levels during the cell cycle in cells of different sizes, cells were elutriated and arrested in G1 for different amounts of time. Samples were then collected during the synchronous progression from G1 through S, G2 and M phases of the cell cycle for RNA extraction and sequencing

592 (See Fig. S2 for schematic of experimental design). Specifically, 4L S. cerevisiae (A17896: W303 cdc28-13) were grown in synthetic complete (SC) media with $2 \%$ glucose at $25^{\circ} \mathrm{C}$ to $\mathrm{OD} \sim 0.75$ and then collected on a filter membrane and resuspend in ice-cold SC media (no carbon source). Cells were then sonicated ( $3 \times 20$ s, 3 minutes on ice between sonication cycles) and loaded into a JE 5.0 elutriation rotor fitted for a two-chamber run (Beckman Coulter) in a J6-MI Centrifuge $\left(2.4 \mathrm{krpm}, 4^{\circ} \mathrm{C}\right)$. The elutriation chambers were pre-equilibrated and run with SC media ( $4^{\circ} \mathrm{C}$, no carbon source). The pump speed was gradually increased until G1 cells with minimal debris were being collected. G1 fractions were then collected on a filter and resuspended in $37^{\circ} \mathrm{C}$ conditioned SC media $+2 \%$ glucose in a $37^{\circ} \mathrm{C}$ shaking water-bath (OD 0.1). The $\mathrm{G} 1$ arrest was maintained at $37^{\circ} \mathrm{C}$ until cells reached either 36-39fL (small), 67-69fL (medium) or 129-131fL (large) as determined by Coulter counter (Fig. S2B\&C). When they reached our target size, cells were released from the $\mathrm{G} 1$ arrest. To do this, cells were collected on a filter membrane and resuspended in $25^{\circ} \mathrm{C} \mathrm{SC}$ media $+2 \%$ glucose (OD $~ 0.35$ ). Samples for size measurement by Coulter counter, DNA-content analysis, and RNA-extraction were taken at 10 minute intervals after release with the 0 minute time point being designated as the time point 30 minutes before the onset of DNA replication (Fig. S2D\&E). For small cells, the 0 minute time point was collected 40-50 minutes after the shift to the permissive temperature, for medium cells the

609 large cells the 0 minute time point was collected 0 minutes after shift to the permissive temperature. Two biological replicates were performed.

For DNA-content analysis, $0.4 \mathrm{ml}$ culture was added to $1 \mathrm{ml} 100 \% 4^{\circ} \mathrm{C}$ ethanol and stored at

$6124^{\circ} \mathrm{C}$. Cells were pelleted (13krpm, 2 minutes), washed, and resuspended in 50mM Sodium Citrate $(\mathrm{pH}$ $613=7.2$ ), incubated with $0.2 \mathrm{mg} / \mathrm{ml}$ RNAse A (overnight, $37^{\circ} \mathrm{C}$ ) and then $0.4 \mathrm{mg} / \mathrm{ml}$ proteinase $\mathrm{K}(1$ hour, $61450^{\circ} \mathrm{C}$ ) before addition of $25 \mu \mathrm{M}$ Sytox Green (ThermoFisher Scientific). Cells were then sonicated and 615 DNA-content was analyzed for >10000 events on a FACScan Analyzer (BD Biosciences). For RNA- 
616 extraction $1.5 \mathrm{ml}$ cells were pelleted (13krpm, 30 seconds) and snap frozen in liquid $\mathrm{N}_{2}$. Samples were

617 then thawed in TRI Reagent (Zymo Rearch) and RNA was extracted as described below (RNA

618 extraction and sequencing).

\section{RNA extraction and sequencing}

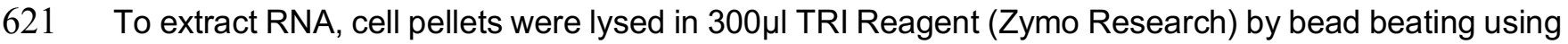
622 a Fastprep $24\left(4^{\circ} \mathrm{C}\right.$, settings: $\left.5.0 \mathrm{~m} / \mathrm{s}, 1 \times 30 \mathrm{~s}\right)$. Cell debris was pelleted $(13 \mathrm{krpm}, 5$ minutes $)$ and the 623 supernatant recovered. RNA was then extracted using the direct-zol RNA microprep kit (Zymo 624 Research). mRNA was enriched using the NEBNext Poly(A) mRNA Magnetic Isolation Module (NEB, 625 E7490) and NEBNext Ultra II RNA Library Prep Kit for Illumina ${ }^{\circledR}$ (NEB, \#E7775) was then used to 626 prepare libraries for paired-end (2x150bp) Illumina sequencing (Novogene). More than 20 million 627 reads were sequenced per sample.

\section{RNA-seq data processing}

630 Because some samples analyzed in this study contained S. cerevisiae as well as reference spike-in S. pombe RNA, a combined S. cerevisiae and S. pombe genome file was created using the sacCer3 and ASM294v2 versions of the respective genomes and a combined transcriptome annotation was

633 created using the S. pombe gene models available from PomBase (Lock et al., 2019) and an S. 634 cerevisiae set of gene models updated using transcript-end mapping data as previously described 635 (Shipony et al., 2020). For the purposes of RNA-seq data quality evaluation and genome browser track 636 generation, reads were aligned against the combined genome and annotated set of splice junctions 637 using the STAR aligner (version 2.5.3a; settings: --limitSjdbInsertNsj 10000000 -638 outFilterMultimapNmax 50 --outFilterMismatchNmax 999 --outFilterMismatchNoverReadLmax 0.04 -639 alignIntronMin 10 --alignIntronMax 1000000 --alignMatesGapMax 1000000 --alignSJoverhangMin 8 640 -alignSJDBoverhangMin 1 --sjdbScore 1 --twopassMode Basic --twopass1readsN -1) (Dobin et al., 641 2013). Read mapping statistics and genome browser tracks were generated using custom Python 642 scripts. For quantification purposes, reads were aligned as $2 \times 50$ mers in transcriptome space against 643 an index generated from the combined annotation described above using Bowtie (Langmead et al. 644 2009; version 1.0.1; settings: -e 200 -a -X 1000). Alignments were then quantified using eXpress 645 (version 1.5.1) (Roberts and Pachter, 2013) before effective read count values and TPM (Transcripts 646 Per Million transcripts) were then separated for each genome and renormalized TPMs were calculated 647 with respect to the total reads for S. cerevisiae.

648 Differential expression analysis by DESeq2 was performed using technical replicates to 649 compare RNA-seq data from different size bins in the experiment shown in Figures 1D-F and S1 (Love 650 et al., 2014). To calculate the total amount of transcription during the G1 arrest/release RNA-seq time 651 course experiment (Figures $1 \mathrm{~J}-\mathrm{L}$ and S2), TPM values were normalized to the mean for each 
652 experiment and the Area Under the Curve (AUC) for TPM as a function of time was calculated for each

653 time course using the R function auc(type = "spline") from the MESS package.

\section{Classification of size-independent transcripts}

656 To classify transcripts whose expression was independent of cell size, we analyzed data from two 657 experiments: (1) the RNA-seq experiment on size-sorted populations of cells (Fig. 1D-F and S1) and 658 (2) the G1 arrest/release RNA-seq time course experiment (Fig. 2A-D and S2). Two biological

659 replicates of each experiment were performed. Sub-scaling genes were classified as genes that 660 passed the following criteria in both biological replicates of each experiment:

661 (1) At least one pair-wise comparison between the four-size bins has a false-discovery rate (FDR) 662 adjusted $p$-value $<0.01$, a TPM fold-change $>1.5$, and bin 1 TPM $>$ bin 4 TPM.

663 (2) Small cells' TPM Area Under Curve (AUC) > medium cells' TPM AUC > large cells' TPM AUC and 664 TPM AUC fold-change > 1.3. See above (RNA-seq data processing) for details of the AUC calculation.

\section{GO term enrichment}

667 GO term enrichment (Fig. 3A) was performed using the GOrilla GO analysis tool (Eden et al., 2009).

668 Enrichment of size-independent gene transcripts was performed versus a background set of all genes 669 that had a TPM value $>0$ in all RNA-seq samples.

\section{Single Molecule Fluorescence In Situ Hybridization (smFISH)}

672 smFISH was used to image WHI5 and MDN1 mRNAs in single cells. A Whi5-mCitrine tagged strain 673 (DCB099) was used to discriminate pre- and post-Start G1 based on Whi5-mCitrine nuclear 674 localization (Doncic et al., 2011). Early S/G2/M cells were defined as budded cells with a small $(\leq 0.2)$ 675 bud-to-mother volume ratio. Cells were grown at $30^{\circ} \mathrm{C}$ in synthetic complete (SC) media $+2 \%$ glycerol $676+1 \%$ ethanol. Two biological replicates were performed. Each biological replicate contained two technical replicates (i.e., two independent hybridizations to cells from the same culture). In addition, two negative controls were performed regularly where (i) FISH probes were omitted and (ii) a whi5s (MK551-1) strain was analyzed.

The smFISH protocol (detailed below) was optimized based on protocols from multiple prior

682 al., 2008). 45ml cells (OD600 0.2) were fixed with $5 \mathrm{~mL} 37 \%$ formaldehyde and incubated (45 683 minutes, room temperature, rotating). Cells were then pelleted ( $1600 \mathrm{~g}, 5$ minutes) and washed twice

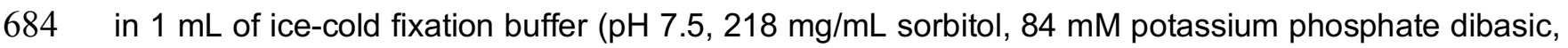
$68516 \mathrm{mM}$ potassium phosphate monobasic, dissolved in water for RNA work (Thermo Fisher Scientific, BP561-1)). Cells were again pelleted and resuspended in $900 \mu \mathrm{L}$ fixation buffer and gently sonicated 
688 BioLabs, S1402S). Cells were then digested by adding 3.5-5 $\mu \mathrm{L}$ zymolyase stock (5 mg/mL 100T, MP

689 Biomedicals, 0832093) and incubated (70-80 minutes, $30^{\circ} \mathrm{C}$, rotating). Cells were then pelleted (400

$690 \mathrm{~g}, 6$ minutes) and washed twice in $1 \mathrm{~mL}$ of ice-cold fixation buffer to stop digestion and finally 691 permeabilized by resuspension in $1 \mathrm{ml} 70 \%$ ethanol. Permeabilized cells were kept at $4^{\circ} \mathrm{C}$ for 1 to 3 692 days. $300 \mu \mathrm{L}$ of the permeabilized cells per hybridization sample were then pelleted ( $400 \mathrm{~g}, 7$ minutes) 693 and washed in $500 \mu \mathrm{L}$ of wash buffer A (Biosearch Technologies, SMF-WA1-60: prepared fresh on 694 the day of use according to manufacturer's instructions always using a fresh aliquot of deionised 695 formamide (EMD Millipore, S4117, stored at $-20^{\circ} \mathrm{C}$ )). Permeabilized cells were then resuspended in $696100 \mu \mathrm{L}$ hybridisation solution (Biosearch Technologies, SMF-HB1-10) containing 1-3x of standard 697 probe concentrations for WHI5 and MDN1 probes, $10 \mathrm{mM} \mathrm{VRC} \mathrm{and} 0.5 \mathrm{mg} / \mathrm{mL} \mathrm{smFISH}$ probe 698 competitor E. coli tRNA (Roche, TRNAMRE- RO). Note VRC and probe competitor were omitted for 699 half the cells analyzed in replicate 1. For MDN1 mRNAs two probe sets, totaling 86 probes $(38+48)$, 700 coupled to the FAM (fluorescein amidite) dye (Biosearch Technologies, Stellaris Custom Probes) were 701 used. The sequences of these probes were taken from Tutucci et al., 2018 (MDN1-3'ORF and MDN1702 ORF). For WHI5, a set of 46 probes coupled to the Quasar570 dye (Biosearch Technologies, Stellaris 703 Custom Probes) were used. WHI5-mCitrine Probe sequences were designed using Stellaris Probe 704 Designer applied to the WHI5-mCitrine mRNA sequence. Probes were hybridised in the dark $\left(30^{\circ} \mathrm{C}\right.$, 705 overnight, with end-over-end rotation). $100 \mu \mathrm{L}$ of wash buffer $\mathrm{A}$ was then added before cells were 706 pelleted $(400 \mathrm{~g}, 8 \mathrm{~min})$ and supernatant was aspirated. Cells were resuspended in $1 \mathrm{~mL}$ wash buffer $707 \mathrm{~A}$, incubated in the dark ( $\left.30^{\circ} \mathrm{C}, 30 \mathrm{~min}\right)$, pelleted ( $\left.400 \mathrm{~g}, 6 \mathrm{~min}\right)$, resuspended in $1 \mathrm{~mL}$ wash buffer $\mathrm{A}$ $708+350 \mu \mathrm{g} / \mathrm{mL}$ calcofluor white (Sigma, F3543), and again incubated in the dark $\left(30^{\circ} \mathrm{C}, 30 \mathrm{~min}\right)$. Cells 709 were then resuspended in $1 \mathrm{~mL}$ wash buffer B (Biosearch Technologies, SMF-WB1-20), incubated for 710 2- 5 minutes at room temperature, pelleted ( $400 \mathrm{~g}, 6$ minutes) and resuspended in 2 to 3 drops ( 75 $711 \mu \mathrm{L}$ ) of Vectashield Antifade Mounting Medium (Vector Laboratories, H-1000). This suspension was 712 then mixed thoroughly by pipetting to separate clumped cells. $1.5 \mu \mathrm{L}$ of this solution was mounted on 713 an acid washed slide and imaged on a wide-field epifluorescence Zeiss Observer Z1 microscope 714 (63X/1.4NA oil immersion objective and a Colibri LED module). 30-step z-stacks (step size = $200 \mathrm{~nm}$ ) 715 were imaged. Cell outlines were identified using phase contrast images. Quasar570 probes (WHI5) 716 were imaged in the orange channel (white LED module for $555 \mathrm{~nm}$ wavelength, $100 \%$ light power, 5 717 sec exposure per stack image). Whi5-mCitrine protein and FAM probes (MDN1) were imaged in the 718 yellow channel (505 nm LED module, $100 \%$ light power, 3.5 seconds exposure time). FAM FISH 719 probes alone were imaged in the green channel (470 nm LED module, $75 \%$ light power, 5 seconds 720 exposure time). Calcofluor white stain was imaged in the blue channel (365 nm LED module, $25 \%$ 721 light power, $20 \mathrm{~ms}$ exposure). Under these conditions, no significant photobleaching was observed 722 after taking multiple images of the same cells. 
smFISH Image analysis was performed manually using ImageJ (version 2.0.0). Single cells were manually selected in each image. A cell was only selected if its morphology was sufficiently intact

725 (following zymolyase treatment) and if the absence/presence of a bud and the nuclear/cytoplasmic 726 localization of Whi5-mCitrine protein could be assigned. Cell size was measured by drawing cell 727 outlines in the phase $z$-plane with the largest cell area, fitting a two-dimensional ellipse, and then 728 rotating the ellipse along its major axis to obtain a volume estimate. Separately calculated volumes for 729 mothers and buds were added together. Absolute counts of WHI5-mCitrine and MDN1 mRNAs in 730 single cells were obtained by manual counting and single dots were counted as one mRNA (i.e., we 731 did not quantify single dot intensities to try to discern multiple overlapping mRNAs). Linear regression 732 was performed in Prism 8 (default settings).

734 Live Cell Microscopy

735 Cells were grown to early log phase in synthetic complete (SC) media $+2 \%$ glycerol $+1 \%$ ethanol 736 and gently sonicated before being loaded into a CellASIC Y04C microfluidics plate (Milipore SIGMA) 737 under continuous media flow at 2 psi. Imaging and pedigree tracking was performed as previously 738 described (Doncic et al., 2013; Schmoller et al., 2015). Cells expressing GFP proteins were exposed 739 for $50 \mathrm{~ms}$ and cells expressing $\mathrm{mCitrine}$ were exposed for $400 \mathrm{~ms}$. Background subtraction for variation 740 in background fluorescence in each frame of the movie was performed as previously describe 741 (Chandler-Brown et al., 2017). Briefly, in each frame cell and non-cell area was defined. A 4-pixel 742 average filter was then applied and the background was taken to be the median filtered pixel value of 743 the non-cell area. Differences in background fluorescence due to cell volume dependent 744 autofluorescence were accounted for as previously described (Schmoller et al., 2015). Note that 745 analysis of WHI5pr-WHI5-mCitrine (Fig. 2F, S3G, 3C\&D) include cells previously imaged, analyzed 746 and reported in Schmoller et al., 2015.

\section{Gene deletion collection screen by microarray}

749 We analyzed the correlation between RNA levels and cell size in 1,484 gene deletion stains using 750 published microarray data and cell size measurements of these strains (Hoose et al., 2012; Jorgensen 751 et al., 2002; Kemmeren et al., 2014; O'Duibhir et al., 2014; Ohya et al., 2005; Soifer and Barkai, 2014). 752 Gene expression changes relative to wild-type were from (O'Duibhir et al., 2014), where we used the 753 dataset transformed to correct for effects of slow growth. The same trends were observed in the 754 uncorrected dataset. For each gene we then analyzed the correlation between then relative fold755 change its expression in a given deletion with the size of that deletion strain across all the deletion 756 strains for which both data were available. Pearson correlation coefficients were calculated using the 757 R function cor (Fig. 3F). 


\section{GFP fusion collection screen by flow cytometry}

760 To examine the size-dependence of an individual protein's expression, we analyzed a genome-wide 761 dataset of flow cytometry-based GFP intensity measurements (Parts et al., 2014), where each 762 measurement is from a single well containing two strains both expressing the same protein C763 terminally fused to GFP. One strain is in the BY4741 background (replicate 1) and the other in the 764 RM11 background (replicate 2). Cells were grown in low fluorescence media containing $2 \%$ glucose 765 and measured using an BD LSRII flow cytometer as described in Parts et al., 2014. Cells were 766 separated into budded and unbudded populations on the basis of the side scatter width (SSC-W). Co767 cultured strains of each background were separated on the basis of HTB2-mCherry intensity (RM low, 768 BY high). Size was defined by the area of the side scatter signal (SSC-A). We used the lowest 769 expressed gene from each plate as a background for that plate, thereby controlling for plate-to-plate 770 variation in measurements. To calculate the background we fitted a linear function to SSC-A and total 771 GFP fluorescence for these low-expressing cells (python function polyfit(matplotlib)). We then 772 subtracted the fit for these lowest expressing cells from the GFP intensity for all other cells. Strains 773 with noisy signals (i.e., their mean expression is less than the standard deviation) and cells with 774 saturated signals (mean expression is greater than 200000) were excluded. SSC-A and background 775 subtracted GFP intensity were then normalized to the mean and a linear function was then fitted 776 (python function polyfit(matplotlib)). The slope of this function was used as a measurement for a 777 protein's size-dependence.

\section{ChIP-seq experiments}

780 Cells expressing Swi4-V5, Swi6-V5, 3xFLAG-WHI5, 3xFLAG-WHI5, GFP-NLS-5xFLAG or Lacl-GFP-

781 NLS-5xFLAG were grown in SC media with $2 \%$ glycerol $1 \%$ ethanol. $500 \mathrm{ml}$ of cells at OD $\sim 0.5$ were 782 fixed with $1 \%$ formaldehyde (30 minutes) and quenched with $0.125 \mathrm{M}$ glycine ( 5 minutes). Fixed cells 783 were washed twice in cold PBS, pelleted, snap-frozen and stored at $-80^{\circ} \mathrm{C}$. Cell lysis and ChIP 784 reactions were performed as previously described (Hu et al., 2015) with minor modifications. Pellets 785 were lysed in $300 \mu \mathrm{L}$ FA lysis buffer (50 mM HEPES-KOH pH 8.0, $150 \mathrm{mM} \mathrm{NaCl}, 1 \mathrm{mM}$ EDTA, 1\% 786 Triton X-100, 0.1\% sodium deoxycholate, $1 \mathrm{mM} \mathrm{PMSF}$, Roche protease inhibitor) with $\sim 1 \mathrm{~mL}$ ceramic 787 beads using a Fastprep-24 (MP Biomedicals). The entire lysate was then collected and adjusted to 1 $788 \mathrm{~mL}$ before sonication with a 1/8' microtip on a Q500 sonicator (Qsonica) for 15 minutes (10 seconds 789 on, 20 seconds off). The sample tube was held suspended in a $-20^{\circ} \mathrm{C} 80 \%$ ethanol bath to prevent 790 sample heating during sonication. Cell debris was then pelleted and the supernatant retained for ChIP. 791 For each ChIP reaction, $30 \mu \mathrm{L}$ Protein G Dynabeads (Invitrogen) were blocked (PBS + 0.5\% BSA), 792 prebound with $10 \mu \mathrm{L}$ anti-V5 antibody (SV5-Pk1, BioRad Cat\# MCA1360G) or $10 \mu \mathrm{L}$ anti-FLAG 793 antibody (M2, SIGMA Ca\#\# F1804) and washed once with PBS before incubation with supernatant 794 ( $4^{\circ} \mathrm{C}$, overnight). Dynabeads were then washed (5 minutes per wash) twice in FA lysis buffer, twice in 
795 high-salt FA lysis buffer (50 mM HepesKOH pH 8.0, 500 mM NaCl, 1 mM EDTA, 1\% Triton X-100,

$7960.1 \%$ sodium deoxycholate, $1 \mathrm{mM}$ PMSF), twice in ChIP wash buffer (10 mM TrisHCl pH 7.5, 0.25 M

$797 \mathrm{LiCl}, 0.5 \%$ NP-40, 0.5\% sodium deoxycholate, $1 \mathrm{mM}$ EDTA, $1 \mathrm{mM}$ PMSF) and once in TE wash buffer

798 (10 mM TrisHCl pH 7.5, $1 \mathrm{mM}$ EDTA, $50 \mathrm{mM} \mathrm{NaCl})$. DNA was eluted in ChIP elution buffer (50 mM

799 TrisHCl pH 7.5, $10 \mathrm{mM}$ EDTA, $1 \%$ SDS) at $65^{\circ} \mathrm{C}$ for $15-20$ minutes. Eluted DNA was incubated to

800 reverse crosslinks $\left(65^{\circ} \mathrm{C}, 5 \mathrm{hr}\right)$, before treatment with RNAse $\mathrm{A}\left(37^{\circ} \mathrm{C}, 1\right.$ hour $)$ and then Proteinase $\mathrm{K}$

$801\left(65^{\circ} \mathrm{C}, 2\right.$ hours). DNA was purified using the ChIP DNA Clean \& Concentrator kit (Zymo Research).

802 Indexed sequencing libraries were generated using the NEBNext Ultra II DNA Library Prep kit (NEB,

803 \# E7645), pooled and sequenced on an Illumina HiSeq instrument as paired end 150bp reads

804 (Genewiz, NJ).

\section{ChIP-seq analysis}

807 Demultipexed fastq files were mapped to the sacCer3 assembly of the $S$. cerevisiae genome as 808 2×36mers using Bowtie (v.1.0.1) (Langmead et al., 2009) with the following settings: -v 2 -k 2 -m 1 -809 best --strata. Duplicate reads were removed using picard-tools (v.1.99). Peaks were called using 810 MACS2 (v.2.1.0) (Feng et al., 2012) with the following settings: -g 12000000-f BAMPE. RPM (Reads 811 Per Million) normalized read coverage genome browser tracks were generated using custom-written 812 python scripts.

\section{Cell Cycle and Protein Partitioning Modeling}

815 The cell cycle was modeled as reported in (Chandler-Brown et al., 2017). We simulated the entire cell

816 cycle, where cells grew and divided according to measured growth and cell cycle transition rates. This 817 accounts for cell-to-cell variability. To examine the role of protein partitioning in the overall scaling of 818 protein expression, we simulated the synthesis of a constitutively expressed protein $(p)$ in each cell.

819 Within the model, protein synthesis and partitioning properties were varied. Protein synthesis was 820 modelled as either size-dependent $\left(\frac{d p}{d t}=k V\right)$ or size-independent $\left(\frac{d p}{d t}=k\right)$ and protein partitioning was 821 modeled as either volume-proportional partitioning at cytokinesis $\left(\left(p_{\text {mother }}=p_{\text {total }} \frac{V_{\text {mother }}}{V_{\text {mother }}+V_{\text {daughter }}}\right)\right.$

822 and $\left.\left(p_{\text {daughter }}=p_{\text {total }} \frac{V_{\text {daughter }}}{V_{\text {mother }}+V_{\text {daughter }}}\right)\right)$ or partitioned in the manner empirically measured for Whi5 823 manner where a significant fraction is partitioned by amount $\left(\frac{p_{\text {mother }}}{V_{\text {mother }}}=1.441 \frac{p_{\text {daughter }}}{V_{\text {daughter }}}\right)$. Cells were 824 simulated until a steady-state distribution was achieved and all cells at the last time-point were plotted. 